\title{
The Spitzer Survey of Interstellar Clouds in the Gould Belt. IV. Lupus V and VI Observed with IRAC and MIPS
}

\author{
Loredana Spezzi ${ }^{1}$, Pierre Vernazza ${ }^{1}$, Bruno Merín ${ }^{2}$, Lori E. Allen ${ }^{3}$, Neal J. Evans II ${ }^{4}$, Jes \\ K. Jørgensen ${ }^{5}$, Tyler L. Bourke ${ }^{6}$, Lucas A. Cieza ${ }^{7}$, Michael M. Dunham ${ }^{8}$, Paul M. Harvey ${ }^{4}$, \\ Tracy L. Huard ${ }^{9}$, Dawn Peterson ${ }^{6}$, Nick F. H. Tothill ${ }^{10}$, \& the Gould's Belt Team
}

\begin{abstract}
We present Gould's Belt (GB) Spitzer IRAC and MIPS observations of the Lupus V and VI clouds and discuss them in combination with near-infrared (2MASS) data. Our observations complement those obtained for other Lupus clouds within the frame of the Spitzer "Core to Disk" (c2d) Legacy Survey. We found 43 Young Stellar Object (YSO) candidates in Lupus V and 45 in Lupus VI, including 2 transition disks, using the standard c2d/GB selection method. None of these sources was classified as a pre-main sequence star from previous optical, near-IR and X-ray surveys. A large majority of these YSO candidates appear to be surrounded by thin disks (Class III; $\sim 79 \%$ in Lupus V and $\sim 87 \%$ in Lupus VI). These Class III abundances differ significantly from those observed for the other Lupus clouds and c2d/GB surveyed star-forming regions, where objects with optically thick disks (Class II) dominate the young population. We investigate various scenarios that can explain this discrepancy. In particular, we show that disk photo-evaporation due to nearby $\mathrm{OB}$ stars is not responsible for the high fraction of Class III objects. The gas surface densities measured for Lupus V and VI lies below the star-formation threshold $\left(\mathrm{A}_{V} \approx 8.6 \mathrm{mag}\right.$ ), while this is not the case for other Lupus clouds. Thus, few Myrs older age for the YSOs in Lupus V and VI with respect to other Lupus clouds is the most likely explanation of the high fraction of Class III objects in these clouds, while a higher characteristic stellar mass might be a contributing factor. Better constraints on the age and binary fraction of the Lupus clouds might solve the puzzle but require further observations.
\end{abstract}

Subject headings: stars: formation - stars: pre-main sequence - stars: low-mass - star forming regions: individual (Lupus V and VI)

\footnotetext{
${ }^{1}$ Research and Scientic Support Dept., ESTEC (ESA), Keplerlaan, 1, PO Box 299, 2200 AG Noordwijk, The Netherlands

${ }^{2}$ Herschel Science Centre, ESAC (ESA), P.O. Box 78, 28691 Villanueva de la Cañada (Madrid), Spain

${ }^{3}$ Department of Astronomy, University of Arizona, 933 N Cherry Ave., Tucson AZ 85721-0065, USA

${ }^{4}$ Department of Astronomy, University of Texas at Austin, 1 University Station C1400 Austin, TX 78712-0259, USA

${ }^{5}$ Centre for Star and Planet Formation, Natural History Museum of Denmark, University of Copenhagen, Øster Voldgade 5-7, DK-1350 Copenhagen, Denmark

${ }^{6}$ Harvard-Smithsonian Astrophysical Observatory, 60 Garden Street, Cambridge, MA 02138, USA

${ }^{7}$ Institute for Astronomy, University of Hawaii at
}

\section{Introduction}

Circumstellar disks of gas and dust, which surround the vast majority of young stars, are the reservoirs of material out of which planets may form (Lada \& Adams 1992; Kenyon \& Hartmann 1995; Currie et al. 2009). Their presence can be inferred via spectroscopic and/or photometric

Manoa, Honolulu, HI 96822, USA

${ }^{8}$ Department of Astronomy, Yale University, P.O. Box 208101, New Haven, CT 06520, USA

${ }^{9}$ Astronomy Department, University of Maryland, College Park, MD 20742, USA

${ }^{10}$ School of Physics, University of Exeter, Exeter, EX4 4QL, UK 
observations at infrared wavelengths as emission in excess of the stellar photosphere. The incidence of primordial protoplanetary accretion disks (with inner radii within $0.1 \mathrm{AU}$ of the central star and a strong, optically thick emission) diminishes with age, being very common at ages of less than $1 \mathrm{Myr}$ and very rare at more than $10 \mathrm{Myr}$ (Mamajek et al. 2004). Stars older than $10 \mathrm{Myr}$ usually show no excess emission, meaning no disk, or have an optically thin mid-infrared emission (tracing material between 0.3 and $3 \mathrm{AU}$ around typical low-mass stars) and show little evidence for substantial reservoirs of circumstellar gas, indicating that they are surrounded by debris disks (e.g., Rieke et al. 2005; Dahm 2005; Currie et al. 2008a, b, 2009; Hillenbrand 2008). Because dust from debris disks can be removed by stellar radiation on very short timescales (less than 0.1 $\mathrm{Myr}$ ), the presence of dust requires an active replenishment source from collisions between larger objects (e.g., Burns et al. 1979; Plavchan et al. 2005). Thus, debris disks around young stars are an indication of active planet formation and their ages also place an upper limit on the formation timescale of gas giant planets. Determining the timescale for the disappearance of primordial disks, and the subsequent dominance of debris disks, is then of primary importance (Currie et al. 2009).

The latter goal has been one of the key objectives of the Spitzer Legacy Project "From Molecular Cores to Planet-forming disks" (c2d; see Evans et al. 2003). The c2d observations included partial spatial coverage of the following clouds: i) Lupus I, III and IV (Merín et al. 2008), ii) Cha II (Alcalá et al. 2008), iii) Perseus (Evans et al. 2009a), iv) Serpens (Harvev et al. 2007b), and v) Ophiuchus (Padgett et al. 2008). The results of the c2d survey were combined by Evans et al. (2009a) in order to provide an updated statistical analysis of the global properties of star formation in all these clouds (star formation rates and efficiencies, numbers and lifetimes of YSOs in each infrared class, clustering properties, etc.).

The Gould's Belt (GB) project is a continuation of the c2d project to complete the Spitzer mapping of nearby star-forming regions in the Gould's Belt (Allen et al., in preparation). In this paper, we present results of the IRAC and MIPS observations of the Lupus V and VI clouds. These obser- vations complement those of Merín et al. (2008) for Lupus I, III, and IV, and allow us to provide a quite complete overview of the evolutionary status of the Lupus star-forming complex. The Lupus cloud complex $\left(\mathrm{RA} \sim 16^{h} 20^{m}-15^{h} 30^{m}\right.$ and DEC $\left.\sim-43^{\circ} 00^{\prime}--33^{\circ} 00^{\prime}\right)$ lies between 150 and 200 pc from the Sun (Comerón 2008), at galactic longitudes $334^{\circ}<l<352^{\circ}$ and latitudes $+5^{\circ}<b<+25^{\circ}$ (Krautter 1992). Lupus is one of the main low-mass star forming complexes, with mid M-type pre-main sequence (PMS) stars dominating its stellar population (Hughes et al. 1994). The complex is located in the ScorpiusCentaurus OB association, whose massive stars are likely to have played a significant role in the evolution and perhaps the origin of the complex (Comerón 2008). With an age of $\sim 1.5-4 \mathrm{Myr}$ (Hughes et al. 1994; Comerón et al. 2003), Lupus lies between the epoch when most stars have optically-thick primordial disks ( 1 Myr) and the epoch where stars are mostly surrounded by debris disks ( $\sim 10 \mathrm{Myr})$. The age estimate strongly relies on the adopted distance to each Lupus cloud. Because the Lupus complex is spread over about $50 \mathrm{pc}$ along the line of sight, the derived stellar age should be considered with caution and apparent age spreads up to $10 \mathrm{Myr}$ or more might be due the uncertain location of each cloud between 150 and 200 pc (Comerón 2008). For a detailed review of the Lupus complex we defer the reader to Comerón (2008), Cambrésv (1999), and Teixeira et al. (2005).

We organize our paper as follows. We first describe our Spitzer observations and data reduction followed by a description of the process by which we identify YSO candidates and eliminate field contaminants. We then characterize the global properties of the YSO candidate samples and compare our results with those of earlier studies for the Lupus complex (Merín et al. 2008) and of other star forming regions observed by Spitzer (Evans et al. 2009a). Finally, we discuss possible evolution scenarios consistent with the observed disk properties of the YSO candidates in Lupus V and VI. In Appendix A we present a detailed description of the several criteria used to corroborate the disk properties of the YSOs candidates. 


\section{Spitzer IRAC and MIPS data}

The Lupus V and Lupus VI dark clouds were observed using the Spitzer Space Telescope IRAC and MIPS cameras at 3.6, 4.5, 5.8, 8.0, 24 and 70 $\mu \mathrm{m}$ on 1-8 April and 9 September 2007 as part of the Spitzer GB survey (PI: Lori E. Allen). A $3.82 \mathrm{deg}^{2}$ and $2.88 \mathrm{deg}^{2}$ areas were mapped in Lupus V and Lupus VI, respectively, covering entirely the cloud area where the visual extinction is greater than $\mathrm{A}_{V} \approx 2$ (Figure 11 and 2) in the extinction map reported by Cambrésy (1999). The observations in Lupus V and VI were performed to match the detection and sensitivity limits of the Spitzer c2d survey in Lupus I, III and VI.

The data reduction procedure was essentially identical to the one used for all the c2d data. The IRAC and MIPS images were first processed by the Spitzer Science Center (SSC) using the standard pipeline to produce the Basic Calibrated Data (BCD). The c2d pipeline for IRAC data has been described by Harvey et al. (2006) and Jørgensen et al. (2006), while the pipeline for MIPS data has been described by Young et al. (2005) and Rebull et al. (2007). Using these BCD images, the final source catalogs were produced following four additional steps:

1. Extra bad-pixel masking, image correction for muxbleed, column pull-downs/pull-ups as well as correction for the "first frame" effect in the third IRAC band (Harvey et al. 2006);

2. Mosaicking of the individual frames. This was done with the SSC's "Mopex" software suite (Makovoz \& Marleau 2005), which eliminates detector transient effects and transient sources (like asteroids) from the mosaics;

3. Source location and extraction from the stacked mosaics, using the c2d developed tool c2dphot (Harvey et al. 2006). This software extracts photometry and calculates the uncertainties using digitized point spread functions. It also gives a morphological classification of the extracted sources (point-like or extended);

4. Cross-identification of the sources among the 4 IRAC bands and merging of the final IRAC catalog with the MIPS 24 and $70 \mu \mathrm{m}$ catalogs (Chapman et al. 2007) and with the near-IR $2 \mu \mathrm{m}$ All Sky Survey (2MASS) cata$\log$ (Cutri et al. 2003). For the band merging, we used a $2.0^{\prime \prime}$ match radius among IRAC bands and 2MASS and 4.0" and 8.0" for matching with MIPS bands 24 and $70 \mu \mathrm{m}$, respectively, to account for the larger point spread functions of the MIPS bands.

For further information on the data reduction procedure we defer the reader to the delivery document by Evans et al. (2007). Table 1 summarizes the number of sources detected with signalto-noise ratio of at least 5 in each band in the two surveyed areas in Lupus V and VI. Note that most of the detected objects are background giant or foreground dwarf stars at the brighter flux levels, while extragalactic objects make a substantial contribution to the fainter source counts.

\section{Selection of YSO candidates}

The main goal of the GB observations is the selection of YSO candidates based on their IR excess emission with respect to older field stars. However, the IR colors of many galaxies are very similar to those of YSOs. Thus, a sample of YSO candidates must be corrected for the contamination of background extragalactic objects. To distinguish YSOs from galaxies, we used a combination of 2MASS, IRAC and MIPS color-color (CC) and color-magnitude (CM) diagrams. This method has been developed within the frame of the Spitzer c2d Legacy Survey and has proven to be a success. Indeed, most of YSO candidates selected in Cha II, Lupus III and Serpens have been subsequently observed via spectroscopy and the YSO nature has been confirmed for $\sim 70 \%$ of them (see, e.g., Spezzi et al. 2008; Merín et al. 2008; Oliveira et al. 2009; Cieza et al. 2010).

A detailed review of the selection method can be found in Harvey et al. (2007a, b) . Briefly, the selection method consists in the definition of an empirical probability function which depends on the relative position of a given source in several CC and CM diagrams, where diffuse boundaries have been determined to obtain an optimal separation between young stars and galaxies.

Figures 3 and 4 show the Spitzer CC and CM diagrams used to select the YSO candidates in 


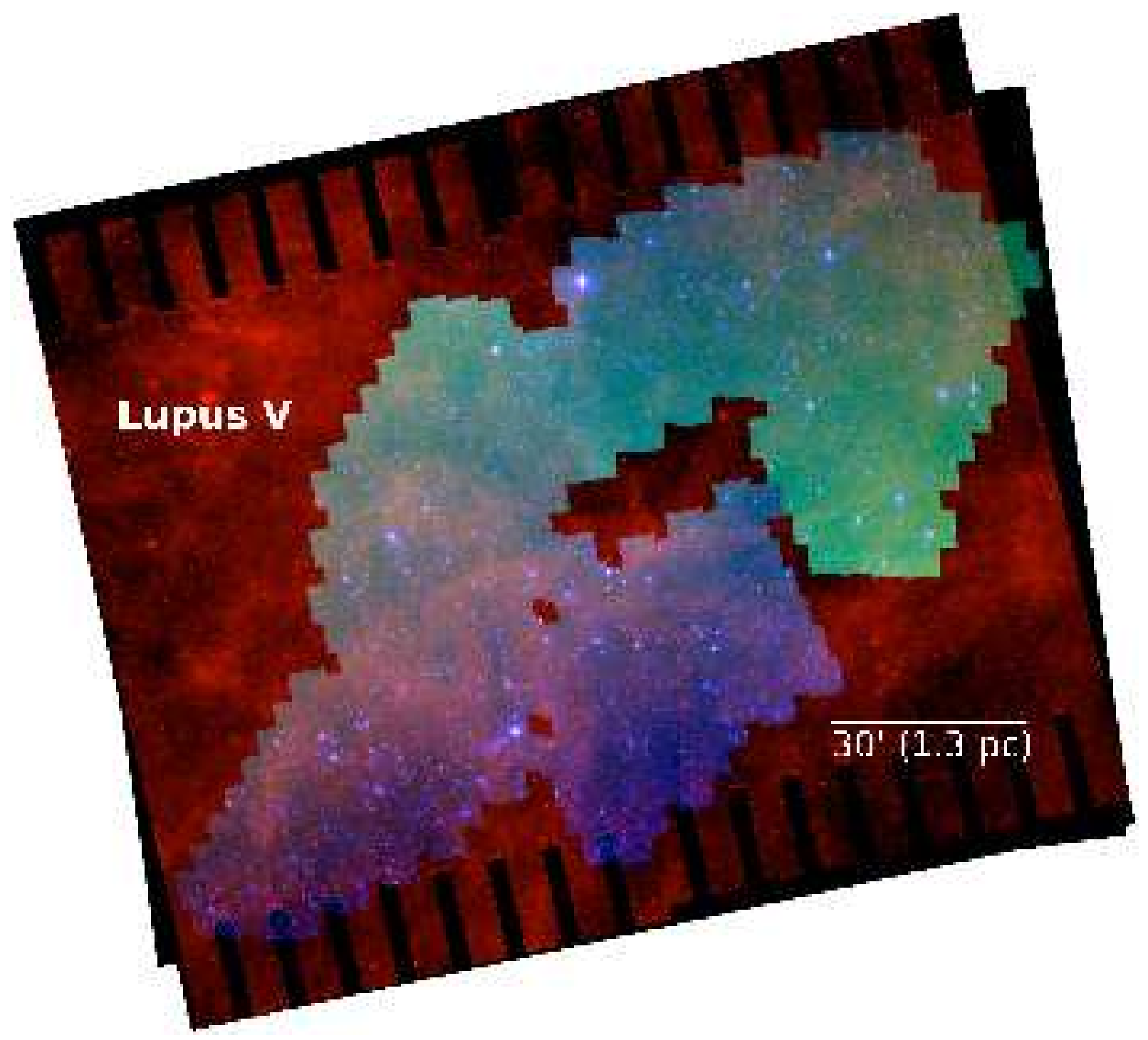

Fig. 1. - Color-composite image of the area in Lupus V mapped by the GB survey. North is up and East to the left. The color code is blue for the IRAC 2 band at $4.5 \mu \mathrm{m}$, green for the IRAC 4 band at $8 \mu \mathrm{m}$, and red for the MIPS 1 band at $24 \mu \mathrm{m}$; note that the IRAC 2 and IRAC 4 mosaics cover exactly the same sky area. The figure shows the remnant cloud structure emitting at long wavelengths. 


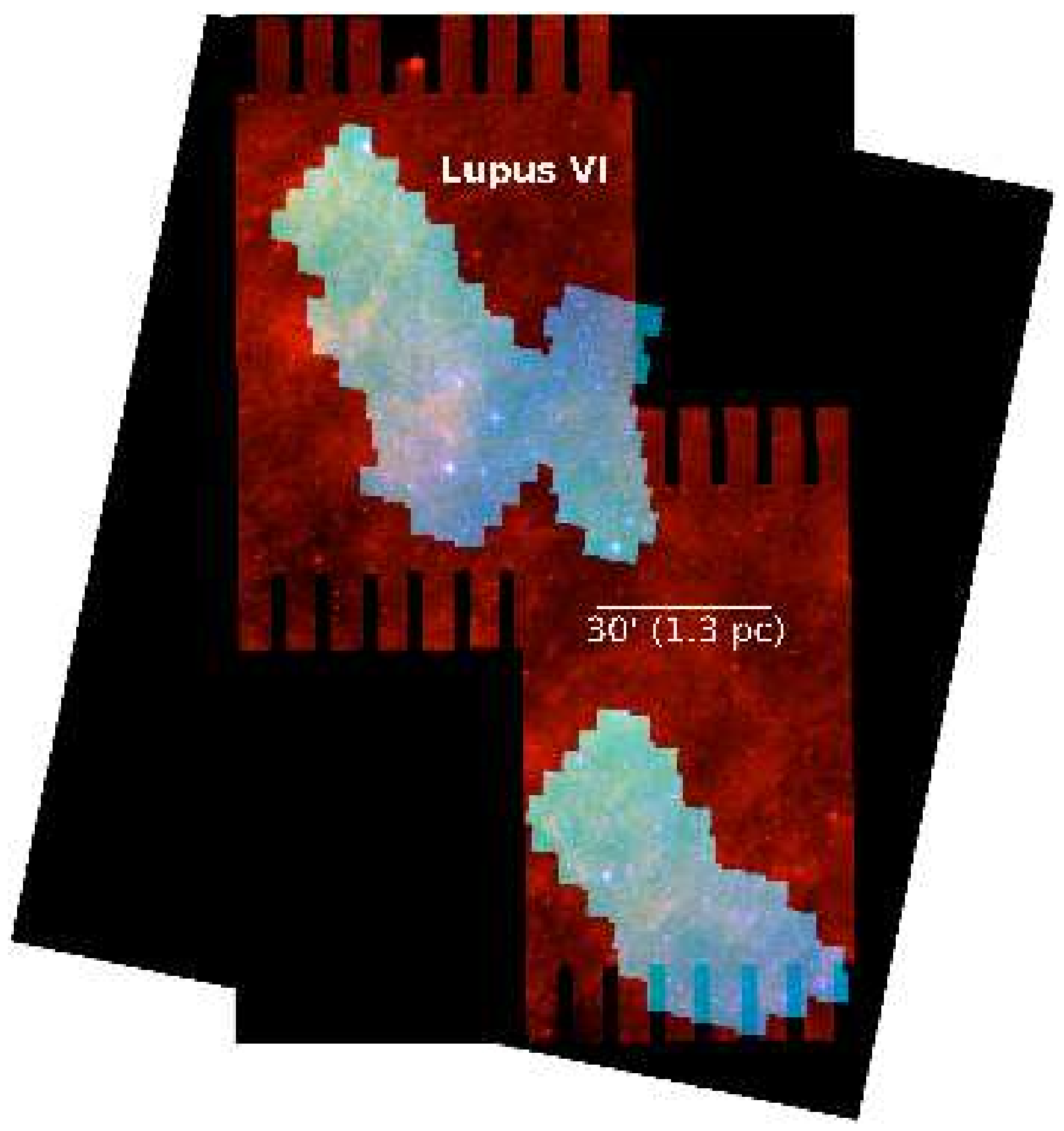

Fig. 2.- Same as Figure 1 for Lupus VI. 
Lupus V and VI, respectively. Note that the method requires detection in all IRAC bands and in MIPS1 with a $\mathrm{S} / \mathrm{N}$ higher than 3 to classify an object as a YSO candidate or a background galaxy. Moreover, older field objects with no IR excess emission are rejected a priori because their IR colors are comparable with normal photospheric colors (e.g., $K-4.5<-0.1$; Harvey et al. 2007a). We find 43 YSO candidates in Lupus $\mathrm{V}$ and 45 in Lupus VI, shown in Figures 3 and 44 as red dots and crosses for point-like and extended sources, respectively. All the YSO candidates have been visually inspected in the IRAC and MIPS images; they appear point-like in all our images and their photometry is not contaminated by crowding, nearby saturated stars or any other artifact that might affect our selection criterion. A remaining caveat is that our YSO candidates might be members of binary/multiple systems too close to be resolved with IRAC/MIPS and, hence, affecting the measured photometry. The multiplicity fraction for low mass stars $(0.5$ $\left.1 \mathrm{M}_{\odot}\right)$ is estimated to be between $20 \%$ and $40 \%$, depending on the actual mass of the primary star and the separation range (Duquennov \& Mavor 1991; Mason et al. 1998; Basri \& Reiners 2006; Lada 2006). Higher resolution photometry or spectroscopy would be needed to assess the actual multiplicity fraction in Lupus V and VI. The effect of multiplicity on the disk properties of YSOs are discussed in Sect. 5.2 .

Interestingly, none of these 88 sources in Lupus $\mathrm{V}$ and VI was classified as a pre-main sequence star from previous ground-based optical/nearIR observations and none of them was detected by the ROSAT X-ray survey (Krautter et al. 1997) or by the IRAS satellite. This "nondetection/classification" is most likely due to photometric incompleteness and lack of dedicated observations for these two clouds. Indeed, no deep optical/near-IR surveys, comparable to those conducted in other Lupus clouds (Comerón et al. 2009), are available so far for Lupus V and VI. Prior to our Spitzer GB observations, only 5 farIR sources were identified in these two clouds by the IRAS satellite (see Table 5 by Comerón 2008). As for X-ray surveys, the ROSAT all sky survey detected about 200 weak-line $\mathrm{T}$ Tauri stars in the Lupus complex mainly concentrated around $\mathrm{Lu}-$ pus III (Krautter et al. 1997). Only an handful of them $(\sim 10)$ are associated with Lupus V and VI, but none of these X-ray sources overlaps with our YSO candidates. No dedicated X-ray observations with XMM or Chandra have been conducted in these two clouds.

In Table 2 3 we list IRAC/MIPS fluxes of the YSO candidates selected in Lupus V and VI, while in Table 4,5 we report their 2MASS magnitudes.

\section{Properties of the YSO candidates}

\subsection{Spectral Energy Distributions and IR Class}

For each of the YSO candidates selected in Lupus $\mathrm{V}$ and VI we constructed the spectral energy distribution (SED), which can be used to constrain specific physical properties of the YSO population to be compared with those of other young populations observed with Spitzer. For all objects, the GB catalog provides the 2MASS near-IR magnitudes and the IRAC $3.6-8 \mu \mathrm{m}$ and MIPS 24$70 \mu \mathrm{m}$ fluxes. We complemented these data with optical photometry (BVRI) from the NOMAD (Zacharias et al. 2005) and DENIS (The 2005) catalogs, thus obtaining complete SEDs from optical to infrared wavelengths depending on available data. Figures 5] show the SEDs for all YSO candidates in Lupus $\mathrm{V}$ and Lupus VI. In each plot we also show, only for visual comparison purposes, the NextGen model spectrum corresponding to a $\mathrm{K} 7$ stellar photosphere (Hauschildt et al. 1999) normalized to the J-band dereddened flux. To perform this comparison, we roughly estimate the visual extinction toward each YSO candidate by fitting the observed photometry between $\mathrm{V}$ and $\mathrm{J}$ with the NextGen model spectrum, arbitrarily assuming a K7 spectral type and $\log g=4$, e.g. the typical values of T Tauri stars in Taurus (see Hartmann et al. 2005). The dereddened flux at each pass-band was obtained adopting the extinction law by Weingartner \& Draine (2001) with $R_{V}=5.5$, also adopted for other Lupus clouds (Merín et al. 2008). Each plot in Figures 5y 8 also shows, for comparison, the typical SED of an optically thick accreting disk, i.e. the median SED of T Tauri stars in Taurus (D'Alessio et al. 1999) normalized to the dereddened J-band flux. From these comparisons, it is clear that the SEDs of our YSO candidates show considerable variety. An ideal classification system has to rely on disk mod- 

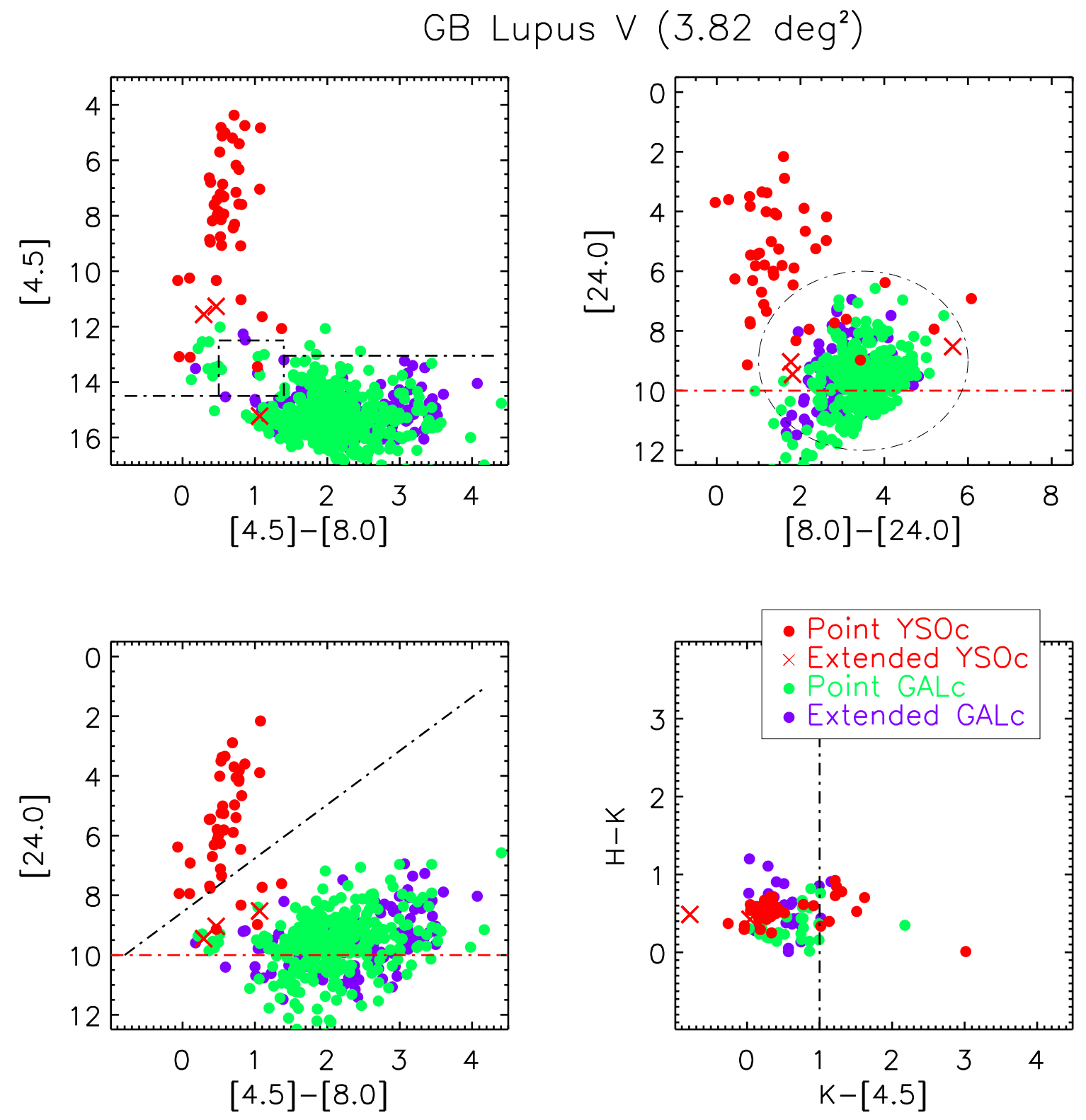

Fig. 3.- CM and CC diagrams for Lupus V. The black dot-dashed lines show fuzzy limits with exponential cut-offs that defines the YSO candidate (YSOc) selection criterion in the each diagram, excluding contamination from galaxy candidates (GALc). Field stars presenting normal photospheric colors are not plotted. The red dot-dashed lines show hard limits, fainter than which objects are excluded from the YSO category. Symbols are as in the legend. 

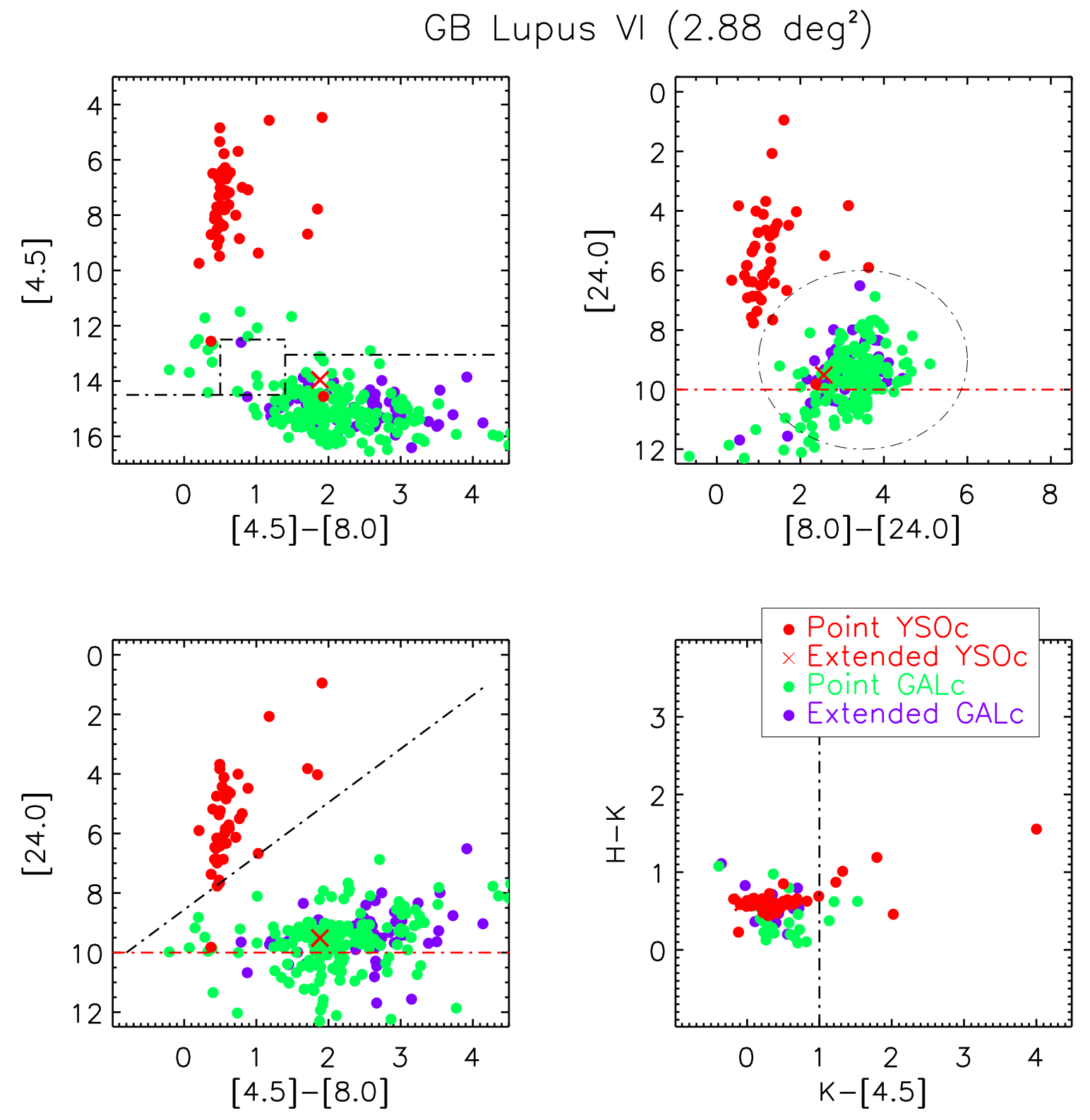

Fig. 4.- Same as Figure 3 for Lupus VI. 
els, which are degenerate in several parameters (Robitaille et al. 2007), and also requires a good knowledge of the physical parameters of the central star (spectral type, reddening, etc), which are not available from the literature for our YSO candidate sample. Therefore, we do not attempt a full disk characterization of each SED but rather classify our objects on the basis of several SED slopes, following the approach adopted in other c2d/GB papers (Harvev et al. 2007a; Merín et al. 2008; Alcalá et al. 2008).

We grouped the YSO candidates identified in Lupus V and VI in four IR classes (Class I, Flat, Class II and Class III) following the scheme proposed by Lada \& Wilking (1984) and Greene et al. (1994). Such grouping allows distinguishing between stars with optically thick and optically thin disk:17; overall, such classification gives a good overview of the evolutionary state of YSOs within a cloud. This classification uses the spectral slope $(\alpha)$ of the SED at wavelengths longer than $2 \mu \mathrm{m}$; in our case, we computed the spectral slope of each SED by performing a least squares fit of all available data in the $\mathrm{K}-24 \mu \mathrm{m}$ range and divided our sample in the four classes according to the following criteria: Class I $\Rightarrow \alpha \geq 0.3$, Flat $\Rightarrow$ $-0.3 \leq \alpha<0.3$, Class II $\Rightarrow-1.6 \leq \alpha<-0.3$, Class III $\Rightarrow \alpha<-1.6$. The values of $\alpha$-slope for the YSO candidates Lupus V and VI are reported in Table 6 and 7 . Figure 9 shows the distribution of spectral slopes for both clouds and Table 8 lists the number of sources in each of the four classes. From Table 8 and Figure 9 we can immediately see that both clouds contain a high fraction of Class III objects ( $79 \%$ for Lupus V and $87 \%$ for Lupus VI), while all other clouds observed with Spitzer show a higher abundance of Class II objects (see Sect. 51). We confirm this classification based on the computation of a single slope $(\alpha)$ by applying several alternative methods, which are described in Appendix A. We also stress that the percentages we estimate are lower limits to the actual number of Class III sources in Lupus V and VI, since our selection criteria require a mid-IR excess and, hence, not all Class III sources are recovered,

\footnotetext{
${ }^{1}$ In this paper we use the definition "optically thin disk" to indicate a disk optically thin at most radii to the radiation from the star. This kind of disk is usually associated with a disk-to-stellar luminosity ratio of $L_{\text {disk }} / L_{\text {star }}<0.01$ (Evans et al. 2009b).
}

having them in many cases normal stellar photospheric colors. Indeed, X-ray observations of Cr A revealed a significant larger number of Class III objects in this cloud than selected by the Spitzer GB survey (Peterson et al., submitted).

\subsection{Interesting objects}

\subsubsection{Transition objects}

Transition disks represent a critical benchmark for disk evolution and planet formation models and it is therefore crucial to study their properties on a statistically significant basis.

We searched for possible transition objects among the YSO candidates identified in both Lupus V and VI. We used the method proposed by Muzerolle et al. (2010) and plot the SED slope in the 3.6-5.8 $\mu \mathrm{m}$ interval $\left(\alpha_{3.6-5.8}\right)$ versus the SED slope in the 8-24 $\mu \mathrm{m}$ interval $\left(\alpha_{8-24}\right)$. These slope values are reported in Table 6 and 7 . Figure 10 shows the $\alpha_{3.6-5.8}$ versus $\alpha_{8-24}$ diagram and the dotted lines define the region where transition objects are expected $\left(\alpha_{8-24}>0\right.$ and $\left.\alpha_{3.6-5.8}<-1.8\right)$. We find two potential transition objects, namely ID 3 in Lupus V and ID 19 in Lupus VI, which are marked with an asterisk in Table 647. The transitional nature of these two objects is also supported on the basis of other SED parameters indicating the presence of a inner hole in their disks (see Sect. A.3 and Figure 19).

\subsubsection{Flat sources}

Flat-spectrum sources, whose emission arises from both a disk and an envelope likely possessing a wind-carved cavity (Greene et al. 1994; Evans et al. 2009b), are believed to be the boundary between protostars and pre-main sequence objects. This phase is one of the most interesting in the evolution of YSOs as phenomena such as outflows and mass accretion, which determine the final mass of the star and process the surrounding material, are particularly active.

The YSO candidates sample selected in Lupus $\mathrm{V}$ and VI contains only one flat sources, namely ID 28 in Lupus VI, and its SED in shown in Figure 7 This object in not detected at optical wavelength neither by the NOMAD nor by the DENIS surveys, as expected for young objects still partially embedded, and appears pointlike in all the 2MASS and Spitzer 3.6-24 $\mu \mathrm{m}$ 


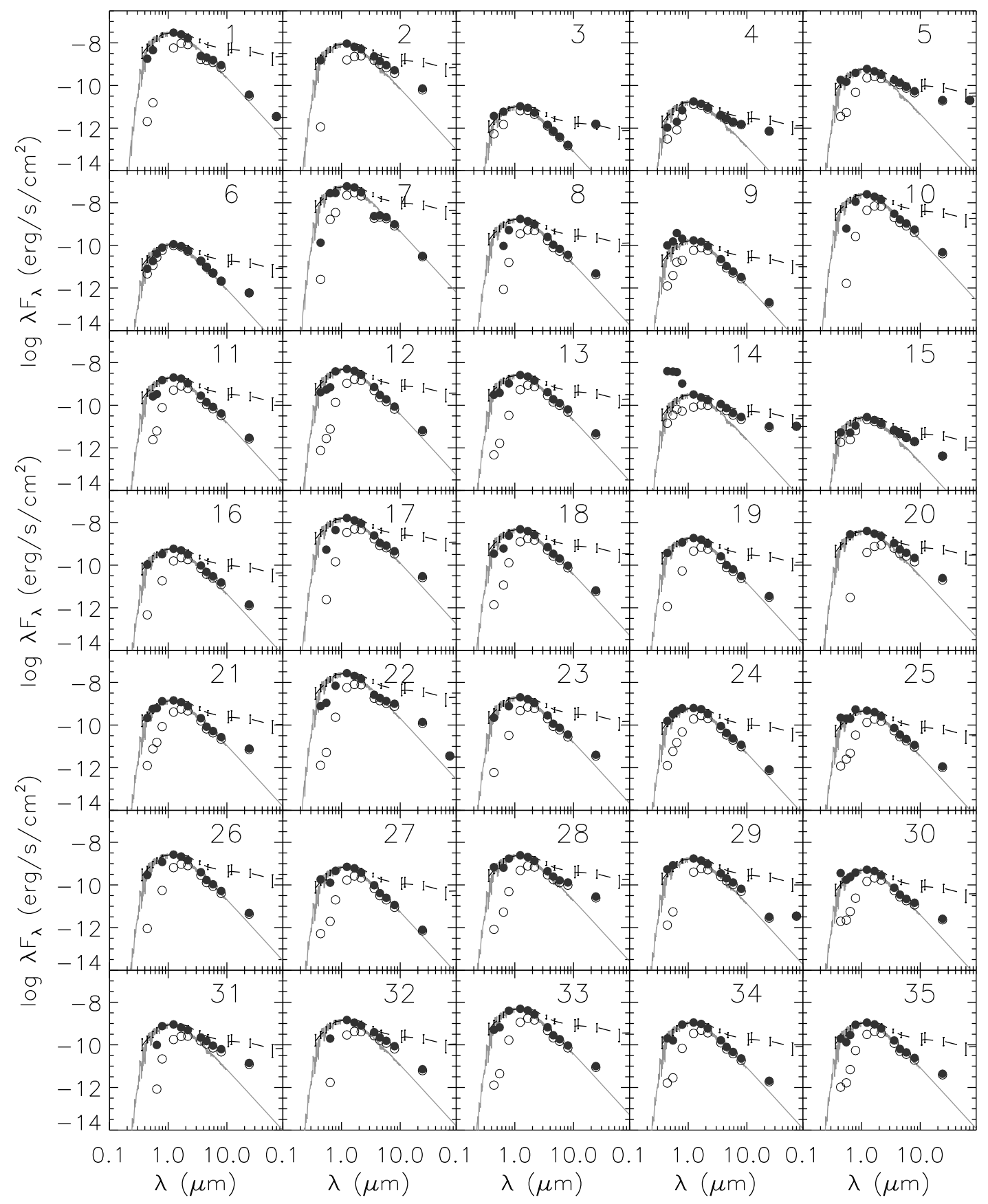

Fig. 5.- Observed (open circles) and dereddened (filled circles) SEDs of the YSO candidates in the Lupus V cloud. The continuous line shows the NextGen photospheric emission model of a K7-type star; the dashed line is the average SED of T Tauri stars in Taurus. Both these reference models are normalized to the J-band flux. 


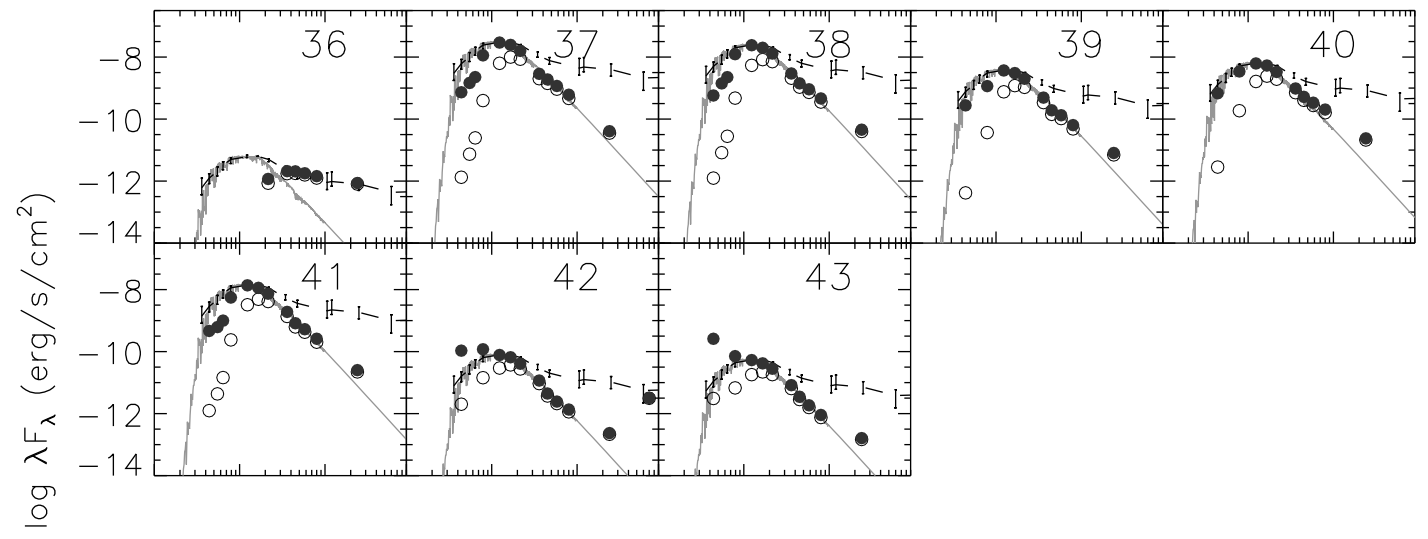

Fig. 6.- Continued. 


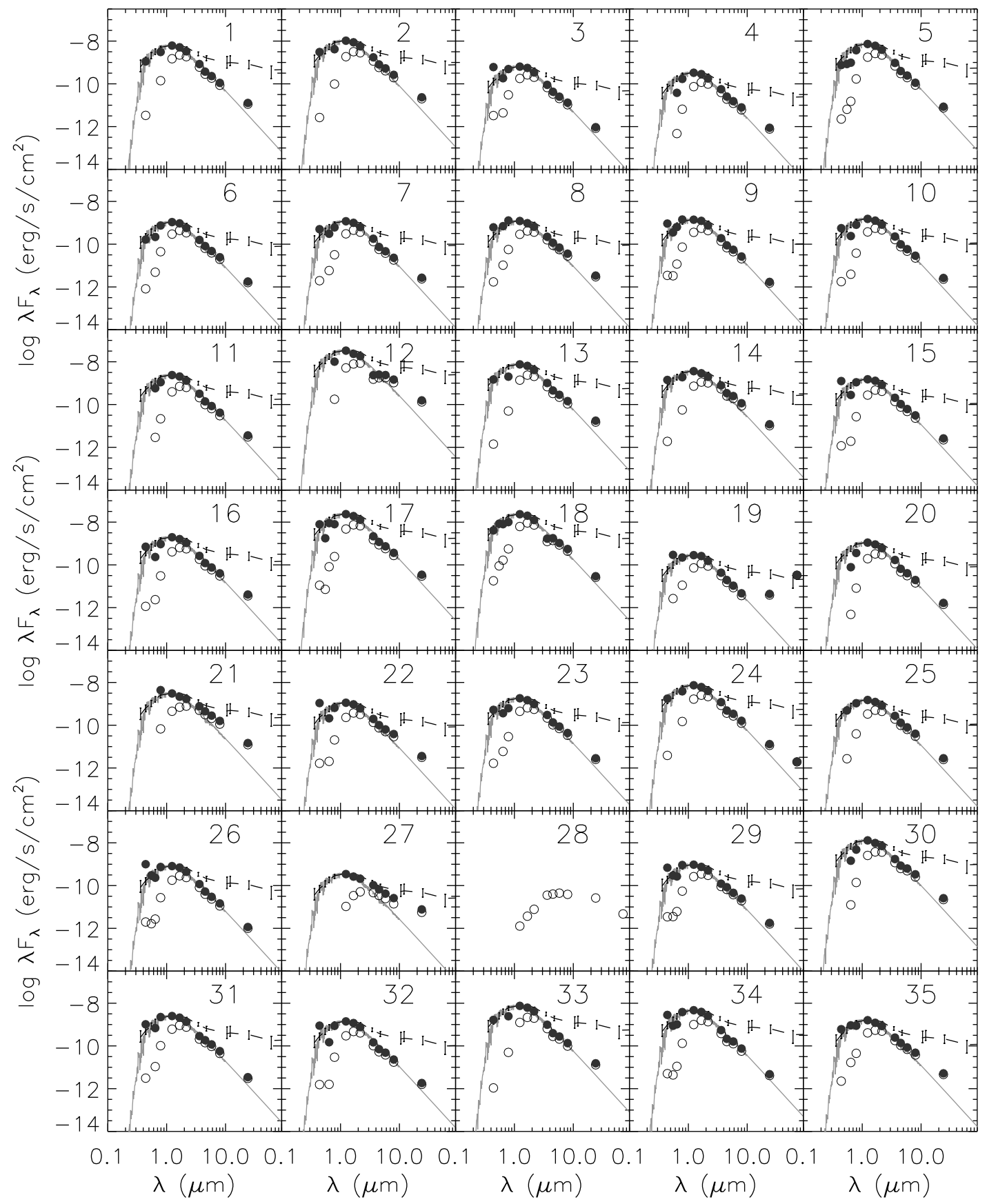

Fig. 7.- Same as Figure 5 for Lupus VI. The flat-spectrum YSO candidate (ID 28) is not detected in the optical and, hence, we do not attempt the comparison with the K7-type stellar model and the the average SED of T Tauri stars in Taurus (see Sect. 4.2.2. 


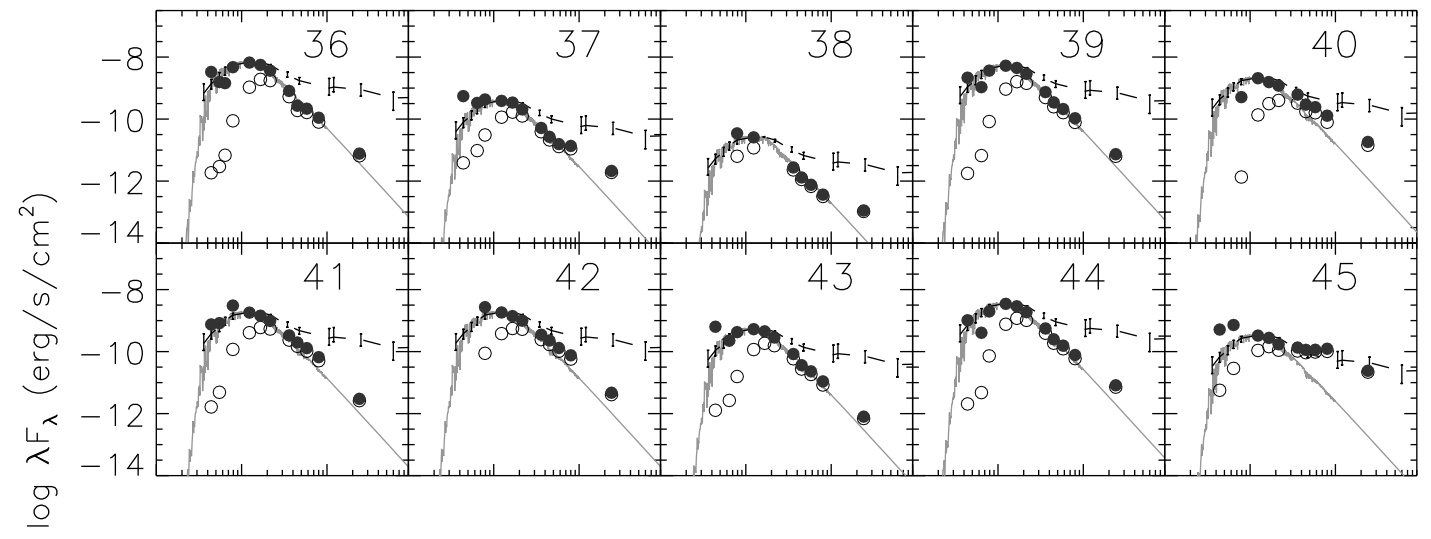

Fig. 8.- Continued. 


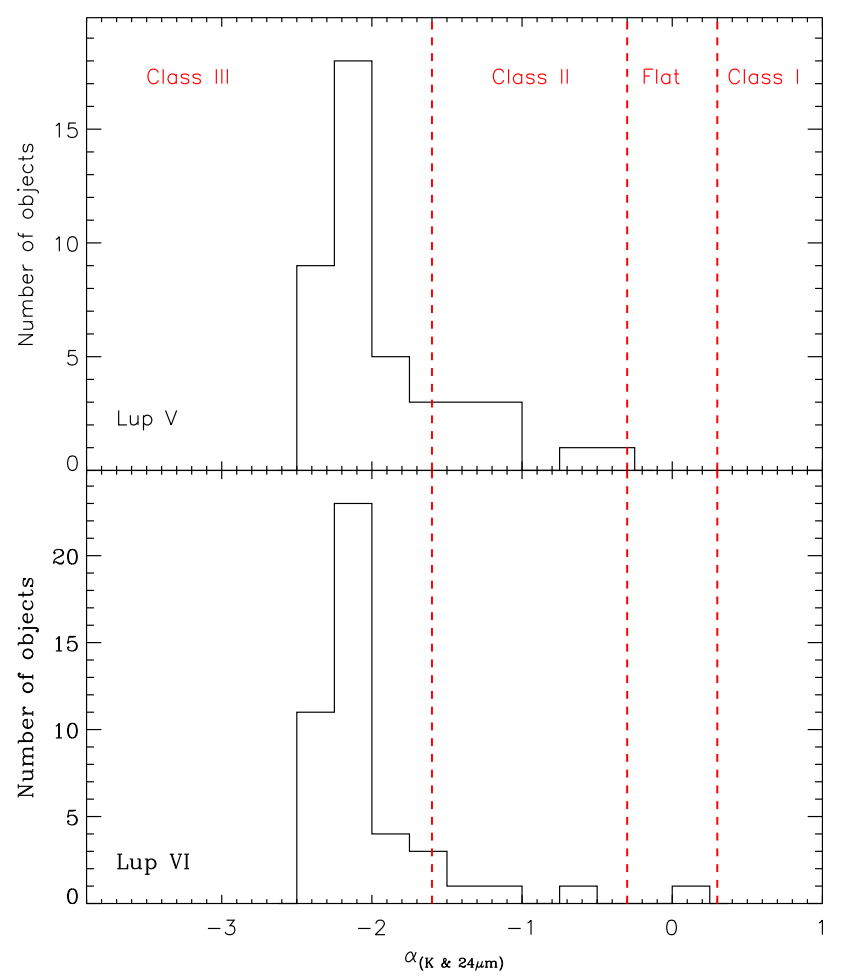

Fig. 9.- $\alpha_{K-24}$-slope distribution of YSO candidates in Lupus V and Lupus VI (solid line). The vertical dashed lines indicate the intervals defining the four Lada classes. Both populations are largely dominated by Class III objects.

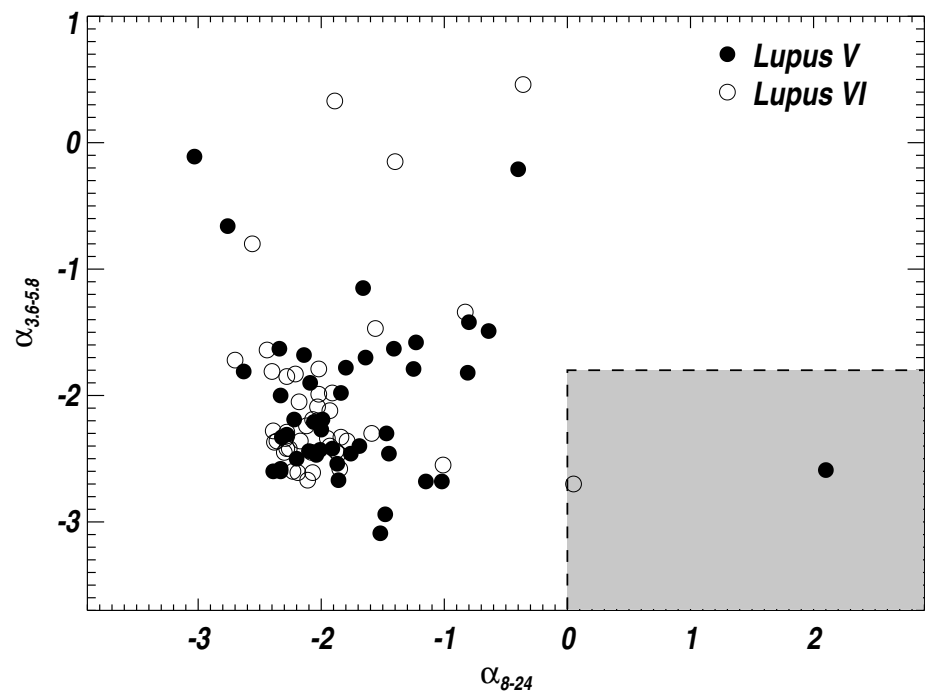

Fig. 10.- SED slope $\alpha_{3.6-5.8}$ as a function of the $\alpha_{8-24}$ slope for YSO candidates in Lupus V and VI. The dashed lines define the region where transition objects are expected $\left(\alpha_{8-24}>0\right.$ and $\left.\alpha_{3.6-5.8}<-1.8\right)$. 
images (Figure 111). This source was recently observed, and not detected, in the $\mathrm{HCO}^{+}(3-2)$ sub-millimeter line by Amanda Heiderman (private communication) using the $10 \mathrm{~m}$ Caltech Submillimeter Observatory (CSO) in Mauna Kea (Hawaii). van Kempen et al. (2009) presented a YSO classification method based on $\mathrm{HCO}+$ lines that successfully separates embedded Class I sources (strong $\mathrm{HCO}+$ emission) from edge-on Class II disks and confused sources (little or no $\mathrm{HCO}+$ emission). The lack of detection in the $\mathrm{HCO}^{+}(3-2)$ line casts doubt on the existence of a residual dense envelope around our flat-spectrum YSO candidate. The upper limit to its main beam $\mathrm{HCO}+$ line temperature, computed as $2 \sigma_{r m s}$, is $<0.48 \mathrm{~K}$.

\subsection{Spatial distribution and clustering of the YSO candidates}

The spatial distribution of YSOs in relation to the cloud structure can be used to study a very specific aspect of the star formation process, i.e. whether it occurs in a centrally condensed way or there is more than one center of density (see Lada \& Lada 2003, and references therein).

Figures 12 and 13 show the spatial distribution of the YSO candidates in the Lupus V and VI clouds on the relative extinction maps obtained from the Spitzer GB data. These extinction maps have a spatial resolution of 120 arcsec and the details on how such maps were derived are given in the final c2d data delivery document (Evans et al. 2007). In both clouds, the regions of high extinction $\left(\mathrm{A}_{V} \gtrsim 6 \mathrm{mag}\right)$ are rare. The great majority of the YSO candidates (of any SED class) lie in the vicinity of the highest extinction regions, however their position does not correlate with the location of the extinction peaks. A possible explanation is that star formation may have not taken place in these clouds very recently (i.e. more than 5-10 Myrs ago), so that their YSO populations appear now to be dispersed in the surroundings of the extinction peaks. This interpretation would fit with the high abundance of Class III objects in both clouds, but needs to be confirmed by velocity dispersion and age measurements (see Sect. [5).

To investigate quantitatively the clustering properties of the YSOs candidates in Lupus V and VI, we applied Casertano \& Hut (1985) surface/volume density algorithm, following the same approach as for the c2d clouds (see, e.g., Evans et al. 2009a). In particular, the criterion we adopt to identify YSO structures is described in detail by Jørgensen et al. (2008) and divides concentrations of YSOs into "clusters" or "groups". "Clusters" are defined as regions with more than 35 YSOs within a given volume density level and "groups" as regions with less. Clusters and groups can be "loose", if their volume density is higher than $1 \mathrm{M}_{\odot} / p c^{3}$, or "tight" if their volume density is higher than $25 \mathrm{M}_{\odot} / p c^{3}$. Figure 14 shows the result of the clustering analysis for Lupus $\mathrm{V}$ and VI. The YSO density contours are plotted, for comparison, on the extinction map. The YSO candidates are divided up into 4 loose groups, two in each cloud. While the two groups observed in Lupus $\mathrm{V}$ are definitively real, the discontinuous coverage of the IRAC observations in Lupus VI might have biased the algorithm result toward the identification of two separate YSO groups in this cloud. Furthermore, there are some low $\mathrm{A}_{V}$ clumps in the eastern part of the region with no associated YSO candidates; this is simply due to the fact that our Spitzer observations did not cover this part of the sky. Table 9 summarizes the results of the clustering analysis in Lupus V and VI; we report the number of YSO candidates, their Lada class, the cloud mass, the volume and the star formation efficiency (SFE) measured in each of the four loose groups. The cloud mass $\left(\mathrm{M}_{\text {cloud }}\right)$ was determined from the GB extinction map, while the SFE is defined as $M_{\text {stars }} /\left(M_{\text {stars }}+M_{\text {cloud }}\right)$, where the total mass converted into stars $\left(\mathrm{M}_{\text {stars }}\right)$ was estimated assuming an average YSO mass of $0.5 \mathrm{M}_{\odot}$, to be consistent with the estimates in Table 12 by Merín et al. (2008) for the other Lupus clouds, which we use for comparison. However, as we will see in Sect. 5.5, the YSO population in Lupus V and VI might have a characteristic stellar mass between 0.6 and $1.3 M_{\odot}$ depending on the assumed age, higher than the other Lupus cloud and slightly higher than the peak of the standard IMF (e.g., Miller \& Scalo 1979; Chabrier 2001; Kroupa \& Weidner 2005). Thus, in Table 9 we also include SFE calculations assuming the standard form of the IMF by Kroupa \& Weidner (2005).

Lupus V and VI clearly show multiple density structures with separate gas concentrations that evolve independently and a SFE around 3-4\% (as- 


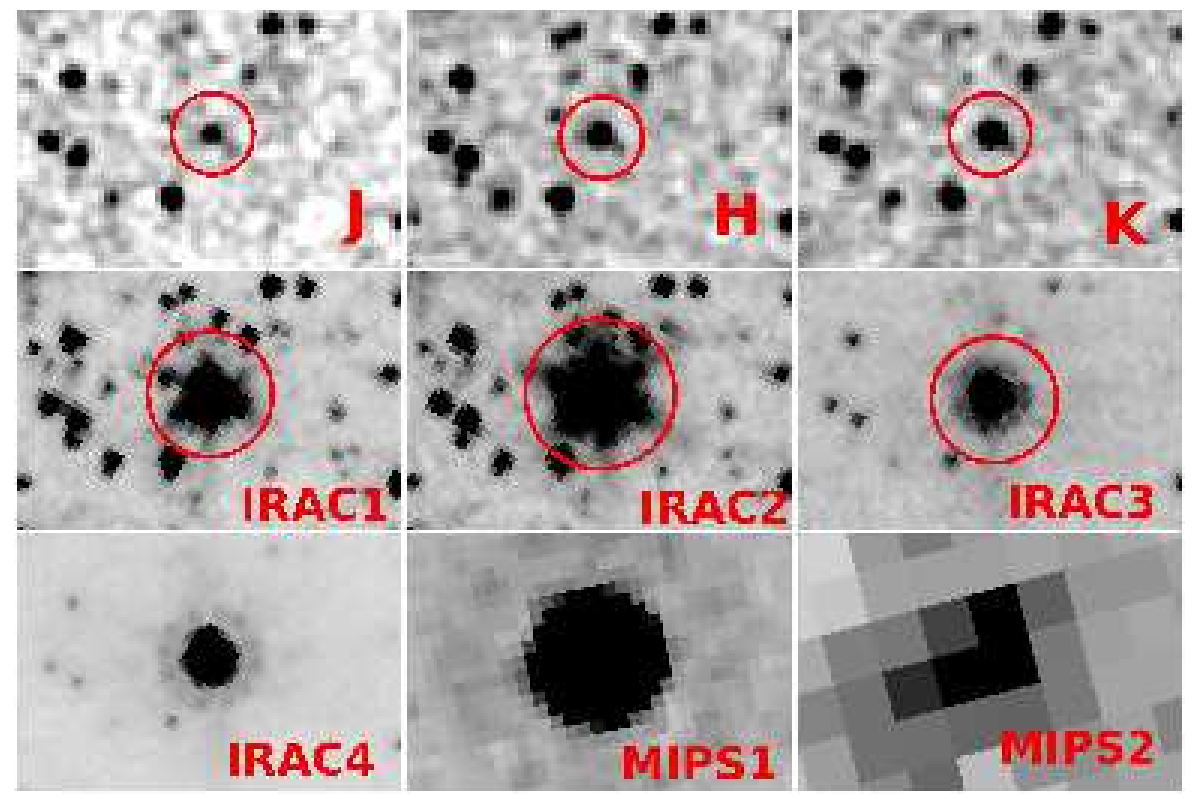

Fig. 11.- 2MASS and Spitzer images of the flat-spectrum YSO candidate identified in Lupus VI (ID 3). Each snapshot covers an area of $75^{\prime \prime} \times 50^{\prime \prime}$; north is up and east to the left.

suming $0.5 \mathrm{M}_{\odot}$ as the typical YSO mass), similar to the SFE measured by Merín et al. (2008) for Lupus I and IV but lower than the SFE measured for Lupus III (see their Table 12). Overall, both the cloud density structure and the distribution of YSO candidates in the two regions suggest a dispersed distribution of volume density enhancements rather than a centrally-condensed structure. This dispersed distribution of YSOs, with peak densities of 2-4 YSO candidates per $\mathrm{pc}^{3}$ following the lane of the dust emission, is typical of Lupus I and IV, while in the more active Lupus III a centrally-condensed structure appears to dominate the star-formation process (Merín et al. 2008). In particular, the clustering analysis shows that the spatial distribution of Class III objects in Lupus V and VI is similar to that of Class III members in Lupus I and IV, while it is clearly more spread than the Class III population in Lupus III. This larger spread might indicate that the Lupus I and IV population is a few Myrs older than the Lupus III population, but again this hypothesis needs to be confirmed by velocity dispersion and age measurements. As we will see Sect. 5, a few Myrs older age (5-10 Myrs) for the stellar population in Lupus V and VI with respect to Lupus III would explain both the higher spatial disper- sion and the higher fraction of Class III objects in these two clouds. Considering this age and given that the YSO candidates in Lupus V and VI are approximately dispersed over a $4 \mathrm{pc}$ radius in both clouds (Figure 14), we expect their velocity dispersion to be of the order of $0.4-0.8 \mathrm{~km} / \mathrm{sec}$, which well matches the range observed for other galactic star forming regions and young clusters (see, e.g., Kraus \& Hillenbrand 2008, and reference therein).

\section{Discussion}

In the previous section we have shown that most YSO candidates in Lupus V (79\%) and Lupus VI $(87 \%)$ are surrounded by optically thin disks (Class III objects). To place our results in a more global context, we report in Table 8 the number of YSO candidates organized by IR class for all the Lupus clouds observed with Spitzer (Lupus I, III, IV, V, and VI; this work and Merín et al. 2008) and the other clouds observed with Spitzer within the frame of the c2d survey, namely Cha II (Alcalá et al. 2008), Perseus (Lai et al., in preparation), Serpens (Harvey et al. 2007b), and Ophiuchus (Allen et al., in preparation). We find a much higher number of the Class III objects in both Lupus V and Lupus VI than in Lupus I, III 


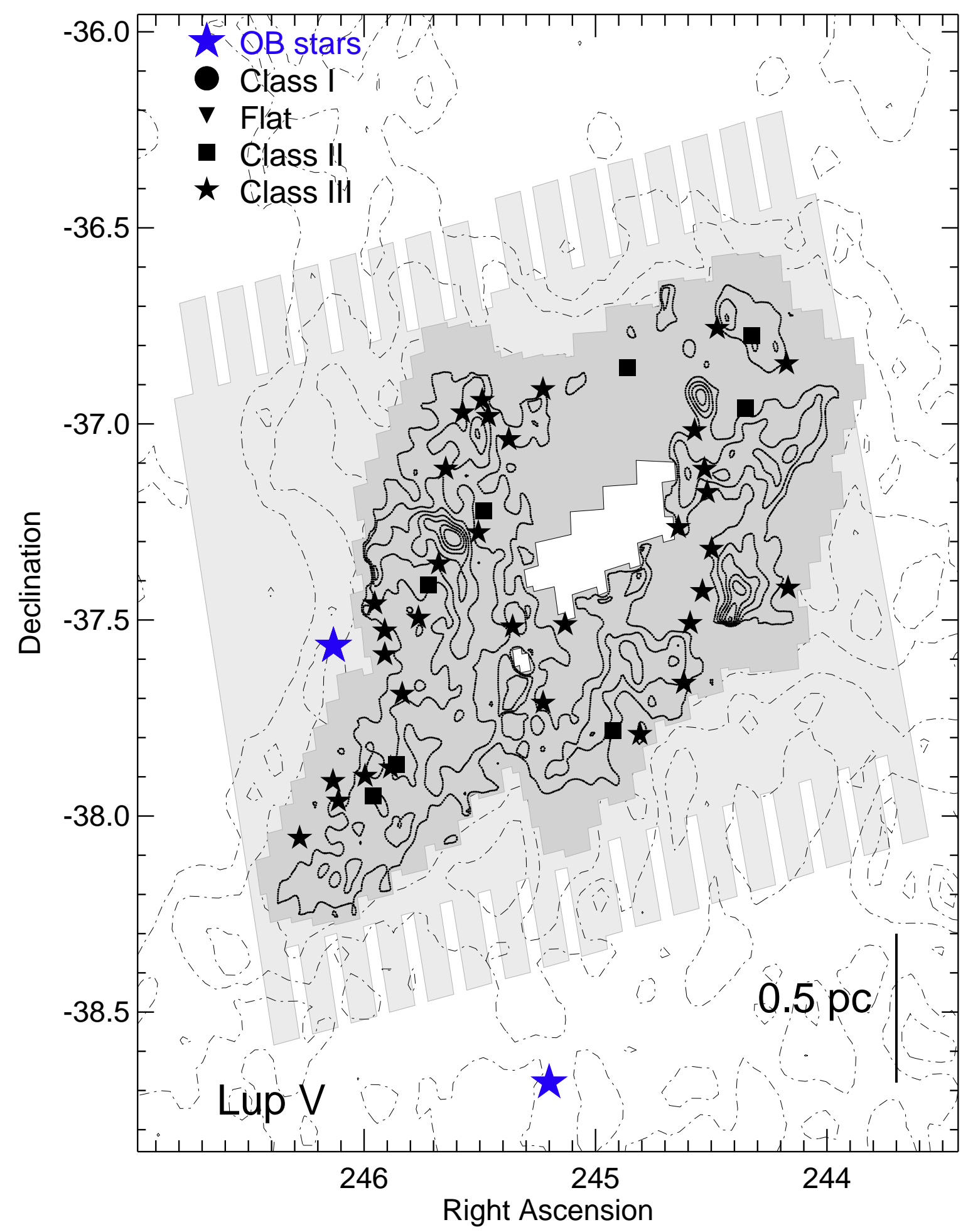

Fig. 12.- Visual extinction $\left(\mathrm{A}_{V}\right)$ contours of the Lupus V cloud, from 2 to 20 mag in steps of 2 mag, from the Spitzer GB extinction map (solid lines). The intersection of the areas observed with the four IRAC bands (dark grey) and the area observed with the MIPS 1 band (light grey) are also shown. The dashed lines outside the IRAC area are the contour levels of extinction from Cambrésy (1999), from 1 to 6 mag in steps of 0.35 mag. Symbols represent the location oflthe YSO candidates in this cloud as a function of the Lada class, as explained in the legend. The position of the OB stars in the field is also indicated. 


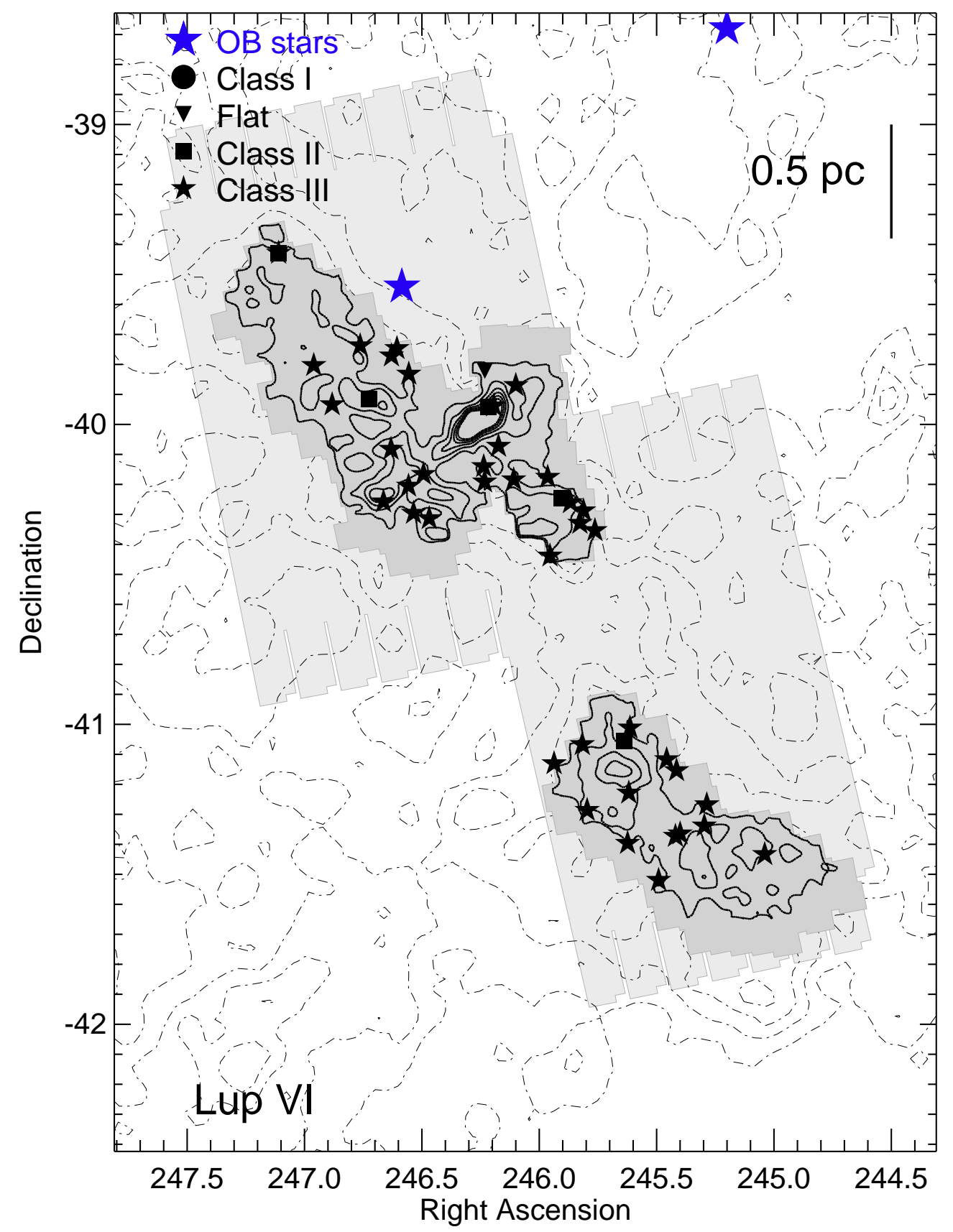

Fig. 13.- Same as Figure 12 for Lupus VI. 


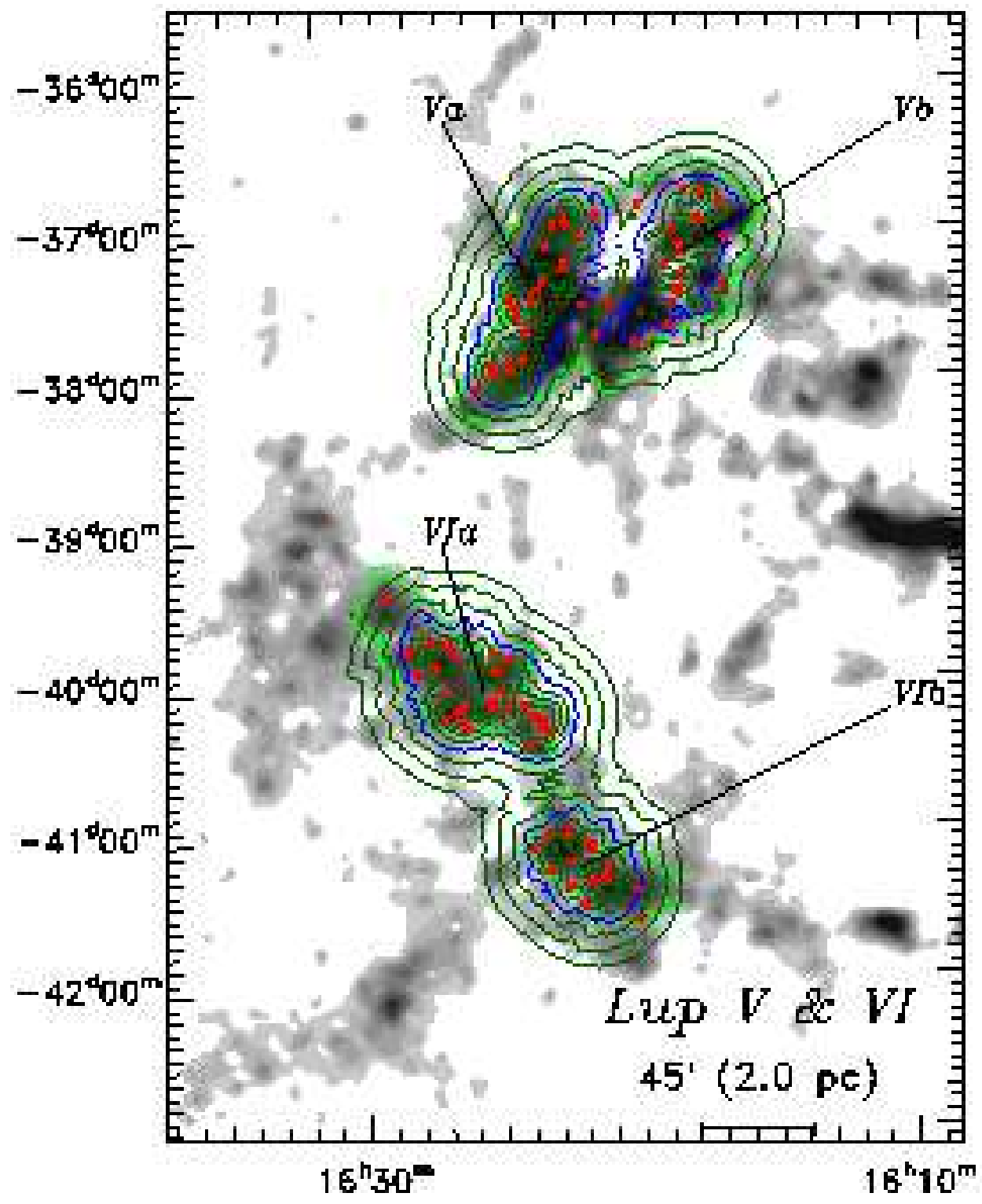

Fig. 14. - Volume density plot for YSO candidates in Lupus V and VI as determined with the nearestneighbor algorithm. The derived density contours are compared with the GB extinction map (in gray scale). The blue contour corresponds to the $1.0 \mathrm{M}_{\odot} / p c^{3}$ density level, while the green ones correspond to levels of $0.125,0.25,0.5,2,4$, and 8 times this level. The four "loose groups" are identified with labels. Red filled circles represent the YSO candidates. The thicker green polygons represent the areas observed with both IRAC and MIPS. 
and IV. The difference gets even stronger when compared to the fraction of Class III objects measured in the c2d cloud sample and summarized in Evans et al. (2009a). To explain the origin of the surprisingly high fraction of Class III objects in Lupus V and Lupus VI, we considered the following possible scenarios and investigate them in more details in Sect. 5.15.5.

1. Contamination: Contamination by field objects with small IR excess emission can affect the measured fraction of Class III YSO candidates.

2. Binarity: Most circum-binary disks are cleared by ages of 1-2 Myr, while most circumstellar disks are not (Bouwman et al. 2006). Thus, a significantly higher fraction of binary stars in Lupus V and VI with respect to the other Lupus clouds would explain the higher fraction of Class III sources.

3. Stellar age: Because Class II objects are very common at ages $\sim 1 \mathrm{Myr}$ and very rare at $\sim 10 \mathrm{Myr}$ (Sect. 1), a few Myrs older age for the stellar population in Lupus V and VI with respect to the other Lupus clouds (1.54 Myr; Hughes et al. 1994; Comerón et al. 2003 ) would also explain the higher fraction of Class III objects.

4. Disk photo-evaporation: This scenario would rely on the presence of OB stars lying close enough to the Lupus V and VI YSO populations to photoevaporate their disks, but too far from the Lupus I, III and IV populations to have any influence on the evolution of their disks.

5. Characteristic stellar mass: Recent studies suggest a much shorter disk dissipation time (Brown et al. 2007; Kim et al. 2009; (Merín et al. 2010) and higher mass accretion rate for higher mass stars (Natta et al. 2004, 2006; Sicilia-Aguilar et al. 2006). Thus, a possible difference between the characteristic stellar mass in Lupus V and VI and the other Lupus clouds may be the cause of the different evolutionary stages of the observed disks.

Using our dataset and complementary information from the literature we now investigate these scenarios.

\subsection{Contamination}

The immediate explanation would be that our YSO candidates sample is significantly contaminated by field objects with small IR excess emission similar to that of Class III YSOs. The adopted YSO selection method has proven to be successful since most YSOs selected in the c2d clouds have been confirmed via spectroscopy (Spezzi et al. 2008; Merín et al. 2008; Oliveira et al. 2009; Cieza et al. 2010). The removal of extra-galactic objects is optimal and the fraction of contaminants, mainly consisting of AGB stars with small excess emission in IRAC and/or MIPS $24 \mu \mathrm{m}$ mimicking the behavior of Class III YSOs, is estimated to be around 30\% (e.g. Oliveira et al. 2009; Cieza et al. 2010). This estimate is based on the spectroscopic follow-up of Class III objects in the Serpens molecular cloud, which is located at $\mathrm{b} \approx+5$ deg from the galactic plane. Thus, we expect the higher galactic latitude of Lupus V and VI $(6<b<8 \mathrm{deg})$ to minimize this problem. However, even taking a $30 \%$ contamination level into account, the fraction of Class III YSO candidates in Lupus V and IV would reduce to $70 \%$ and $81 \%$, respectively, and is still much higher than measured in other c2d clouds.

To verify further the amount of expected contamination, we have also estimated the numbers of background AGB stars expected towards the direction of Lupus V and VI over the areas observed with Spitzer. We performed this exercise by using the Galaxy model by Robin et al. (2003) and their online too 2. According to this model, we expect less than 1 background AGB stars in the 3.82 and $2.88 \mathrm{deg}^{2}$ areas observed in Lupus V and VI, respectively, with apparent $J$ magnitude between 5 and 16.5 , i.e. the photometric ranges covered by our YSO candidates (Tables 4 and 51). Galactic counts models become more and more inaccurate towards the very low-mass regime and the predicted number depends on the adopted model, however this exercise ensures that AGB stars can not contribute noticeably to the contamination of our Class III YSO candidates.

\footnotetext{
${ }^{2}$ http://model.obs-besancon.fr/
} 


\subsection{Binarity}

It has been shown that interactions with close stellar or planetary companions can significantly influence the evolution and lifetime of protoplanetary disks (Monin et al. 2007). In particular, Kraus \& Ireland (2010) have shown that disk evolution of close (5-30 AU) binary systems is very different from that of single stars, and most circum-binary disks are cleared by ages of 1-2 Myrs, while most circumstellar disks around single stars are not (Bouwman et al. 2006). Thus, a significantly higher fraction of binary stars in Lupus V and VI with respect to the other Lupus cloud would also explain the high fraction of Class III sources. Specifically, the fraction of binary stars in Lupus V and VI should be about 50\% higher than in the other Lupus clouds to account for the relative abundance of Class III YSOs.

There are no indications in the literature of a higher binary fraction in Lupus V and VI relative to other Lupus clouds and, hence, this scenario appears unlikely. However, we cannot definitively rule it out. High resolution spectroscopy (see, e.g., Prato 2007) and/or adaptive optics and long-baseline interferometry would be necessary to properly measure the binary fraction in these clouds (e.g., Raghavan et al. 2007).

\subsection{Stellar age}

IR studies of individual star-forming regions have long suggested that disk lifetimes are relatively short ( $\leqslant 5$ Myrs; Strom et al. 1989; Skrutskie et al. 1990; Lada \& Lada 1995; Brandner et al. 2000). Haisch et al. (2001) were the first to provide more precise constraints. They showed that the fraction of stars with disk within a cluster rapidly decreases with age, such that one-half the stars lose their disks within the first $3 \mathrm{Myr}$, and derived an overall disk lifetime of $\sim 6 \mathrm{Myr}$. More recently, Evans et al. (2009a) derived half-life for each of the Lada classes from the combined analysis of the Spitzer c2d dataset. According to this study, the half-life for Class II sources is $\sim 2$ Myrs. Thus, an age of $\sim 10$ Myr for the young population in Lupus V and VI, i.e. only a few Myrs older than the other Lupus clouds (1.5-4 Myr; Hughes et al. 1994; Comerón et al. 2003), would explain the higher fraction of Class III objects in these clouds.
We can not assess the presence of age differences of the order of a few Myrs among the Lupus clouds on the basis of the Spitzer and complementary data available at the moment in the literature. Optical photometry and spectroscopy would help to estimate isochronal ages and/or ages based on the LiI $670.8 \mathrm{~nm}$ line for the YSO population in each Lupus cloud. However, these methods provide only weak constraints on the age (see, i.e., Palla et al. 2005), so that a possible age difference of a few Myrs between the Lupus V and VI population and the population in other Lupus clouds would not be clearly reflected by their LiI $670.8 \mathrm{~nm}$ absorption line or position in optical/near-IR color magnitude diagrams. Moreover, the reliability of lithium abundance as an age indicator is even more limited after the recent finding that events of episodic accretion enhance the lithium depletion of low-mass stars and brown dwarfs (Baraffe \& Chabrier 2010). As for the isochronal age, the uncertainty on the distance to the Lupus clouds prevents a reliable derivation of the age based on the position of the objects in the temperature-luminosity diagram. This problem will be solved in the near future thanks to the forthcoming GAIA mission.

Beside the impossibility to put accurate constraints to the age of Lupus V and VI on the basis of Spitzer data, it is plausible that in these two clouds star formation ceased almost entirely a few Myrs ago while this is not the case for other Lupus clouds. Heiderman et al. (2010) investigate the relation between star formation rate (SFR) and gas surface densities $\left(\Sigma_{\text {gas }}\right)$ for 20 large molecular clouds from the $\mathrm{c} 2 \mathrm{~d}$ and GB surveys. This study revealed that the SFR surface density $\left(\Sigma_{\mathrm{SFR}_{\mathrm{IR}}}\right)$ versus $\Sigma_{\text {gas }}$ relation is a power law whose slope changes from a steep relation at $\Sigma_{\text {gas }}<\Sigma_{t h}$ (slope of $\sim 4.6$ ) to a linear relation (slope of $\sim 1.1$ ) above $\Sigma_{t h}$. The authors estimate $\Sigma_{t h}=129 \pm 14 \mathrm{M}_{\odot} / p c^{2}$, corresponding to $\mathrm{A}_{V} \approx 8.6 \mathrm{mag}$, and denote this as a star formation threshold. This does not imply no star formation below the threshold, but clearly indicates that the probability to find star forming cores is higher above the threshold. The star-forming threshold found by Heiderman et al. $(2010)$ is in agreement with the threshold $\left(\mathrm{A}_{V} \approx 7\right.$ $10 \mathrm{mag}$ ) found in other studies of local molecular clouds (Onishi et al. 1998; Johnstone et al. 2004; Enoch et al. 2007; Lada et al. 2010; André et al. 
2010). In other words, if most of the presentday mass measured for a given cloud lies below $\mathrm{A}_{V} \approx 8 \mathrm{mag}$, a decrease in star formation could plausibly be caused by exhaustion of gas above such a threshold in surface density. In Table 10 we report the fraction of cloud mass below the threshold for Lupus V and VI and compare it with the fraction measured for the other Lupus clouds, in particular for Lupus III which is the most active star forming cloud in the complex and is mainly populated by Class II objects (see Table 8). It is clear that the cloud mass above threshold in Lupus $\mathrm{V}$ and VI is very small or zero, while in Lupus III and in the other Lupus clouds a significant fraction of the cloud mass is still above threshold. Thus, it is not surprising that star formation is still ongoing in Lupus III, as reflected by the substantial number of objects in younger SED classes. In contrast, the absence of such sources in Lupus $\mathrm{V}$ and VI could be explained by a cessation of active star formation a few Myrs ago. It may be hard to get an actual age difference between Lupus V and VI on the one hand and the other Lupus clouds on the other, both for the reasons mentioned above and because the oldest stars in each cloud may have similar ages. Table 10 only indicates that the duration of the star-forming event in Lupus $\mathrm{V}$ and VI was less because it started with less dense gas and used it up longer ago.

Another argument in favor of an older age of Lupus V and VI with respect to other Lupus clouds comes from their spatial location with respect to the Sco-Cen association. Lupus I, III, IV are all at high galactic latitude $(b \approx+9,+16$ and +48 , respectively), in the region of the Lupus complex closest to Sco-Cen (see Figure 15 and Figure 2 by Tachihara et al. 2001). In particular, Lupus I is at the edge of an HI shell left over from a supernova event in the Upper-Sco sub-group of Sco-Cen about 1.5 Myr ago (Tachihara et al. 2001). The dynamics of the $\mathrm{HI}$ and the CO towards Lupus I are consistent with the molecular cloud being compressed by the HI shell (Tothill et al. 2009), and this may have caused recent/ongoing star formation. Lupus III and IV lie in "streamers" that stretch from the main body of Lupus towards the Upper-Cen-Lup sub-group of Sco-Cen, but may also be exposed to effects from Upper-Sco. Thus, even though Lupus III and IV do not fit the argument so well as Lupus I, recent triggered star- formation might play a role in their star formation history, whereas Lupus V and VI lie at lower galactic latitudes $(b \approx+8$ and +6 , respectively) and do not show any morphological evidence of interaction with Sco-Cen.

\subsection{OB stars and disk photoevaporation}

Circumstellar disks in stellar aggregates can be effectively evaporated within a few Myrs through the action of external ultraviolet radiation from the most massive stars in the group. The intensity and spatial extent of such radiation fields depends on the number and spectral type of the massive stars. For small groups of 100/500 members, which is the case of the Lupus complex, photoevaporation of disks around low-mass stars $\left(\mathrm{M} \lesssim 1 \mathrm{M}_{\odot}\right)$ is expected to be efficient within about 0.5-1 pc of the massive stars (see Johnstone et al. 1998; Störzer \& Hollenbach 1999; Adams et al. 2004; Balog et al. 2007; Guarcello et al. 2009, and references therein). Indeed, it has been recently shown that in the young open clusters NGC 2244 and NGC 6611, both containing a rich low-mass PMS population as well as a large number of OB stars, the disk frequency drops within a distance of $\sim 0.5 \mathrm{pc}$ from the massive star group (Balog et al. 2007; Guarcello et al. 2009). At larger distances, however, stars with disks do not appear affected by the presence of massive stars, since the disk frequency is not spatially correlated with the positions of massive stars. We can consequently assume that any disk photoevaporation effect is expected to be seen within $\sim 1$ pc from the ionizing OB stars.

We used the OB star catalog provided by C. Reed (Reed 2003) and searched for OB stars lying in the vicinity, i.e. within $1 \mathrm{pc}$, of the Lupus clouds. Figure 15 shows the spatial distribution of massive OB stars together with the areas observed by Spitzer in Lupus I, III, IV, V and V as part of the $\mathrm{c} 2 \mathrm{~d}$ and GB surveys. In Table 11 we report the number of OB stars within 1 pc of each of the Lupus clouds, their spectral type and luminosity and the projected distance from the cloud. Because of the paucity of OB stars in the vicinity each cloud, it is very unlikely that photoevaporation plays a relevant role in the disk evolution of the YSOs in these clouds. In particular, for Lupus $\mathrm{V}$ and VI we found just one star lying within 1 pc from each cloud, as shown in more detail 


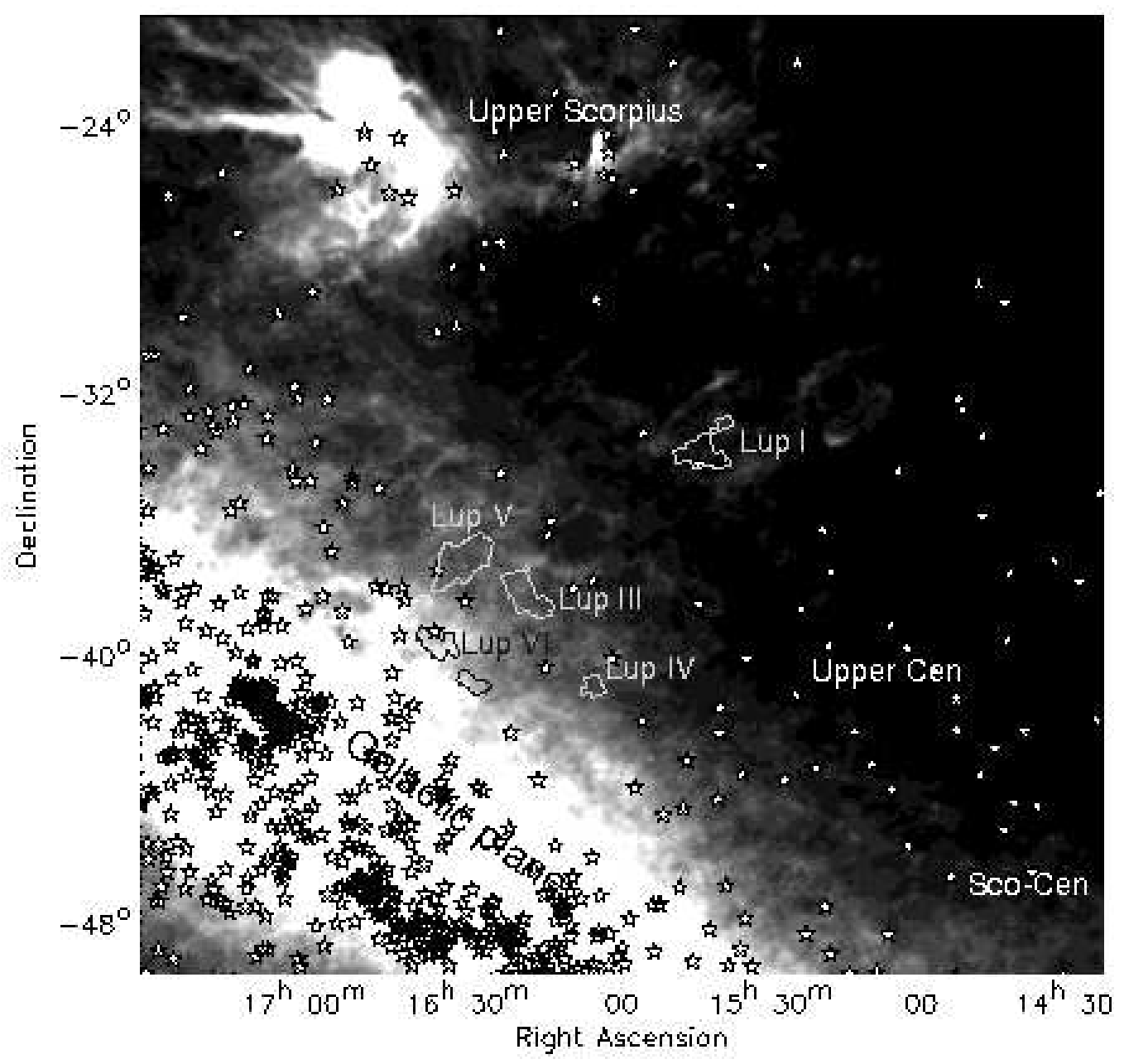

Fig. 15.- IRAS $100 \mu \mathrm{m}$ dust emission map of the Lupus cloud complex from the Galactic Dust Reddening and Extinction database at NASA/IPAC Infrared Archive. The polygons represent the areas mapped with IRAC and MIPS in Lup I, III, IV, V and V as part of the c2d and GB surveys. The stars indicate the position of the OB stars from the catalog by C. Reed (private communication). The positions of the Galactic plane and the Scorpius-Centaurus main OB associations are also indicated. 
in Figure 12 and Figure 13, and this number is comparable to that found in Lupus I, III and IV. Thus, there is no evidence that disk photoevaporation is acting more efficiently in Lupus V and VI then in the other Lupus clouds. Moreover, both for Lupus V and VI, we did not find any spatial correlation between the Class II disk frequencies and the position of these massive stars. For instance, in Lupus VI, Class II YSO candidates are more abundant within $1 \mathrm{pc}$ from the only B-type star in the field than further away. Another argument discharging the photoevaporation hypothesis is that the radius up to which a star can externally photoevaporate a disk depends on the square root of its ionizing flux, which in turn depends on the spectral type (see, e.g., Johnstone et al. 1998; Störzer \& Hollenbach 1999; Adams et al. 2004; Balog et al. 2007; Guarcello et al. 2009). Radii of the order of $0.5-1 \mathrm{pc}$ apply to early and midtype $\mathrm{O}$ stars, which have ionizing fluxes orders of magnitude greater than those of mid or late B stars. As Table 11 indicates, the nearest stars to the Lupus clouds have mid or late B types, and are thus virtually harmless to any nearby disks.

For these overall reasons, it is very unlikely that the proximity of massive stars could be the cause of the high abundance of Class III YSO candidates in Lupus V and VI.

\subsection{Luminosity function and characteris- tic stellar mass}

The determination of the stellar luminosity can be used as a poor but still useful first order proxy for mass, assuming that most of the stars have formed more or less at the same time. Before using the range of luminosities to provide an estimate of the mass range, we determined the degree of completeness of the YSO candidate sample in Lupus V and VI. We used the same approach as Merín et al. (2008) and derive first the bolometric luminosity function for Lupus $\mathrm{V}$ and VI. The bolometric luminosity of each YSO candidate was obtained by integrating over the entire observed SED flux; the total flux was finally converted to luminosity assuming a distance of $150 \mathrm{pc}$ for both Lupus V and VI clouds (Comerón et al. 2009). We then applied Harvey et al. (2007b)'s completeness estimate for the c2d survey to our YSO candidate samples, since the GB catalogs have the same photometric depth as the c2d ones. Harvey et al. (2007b) made an estimate of the completeness of the $\mathrm{c} 2 \mathrm{~d}$ catalogs by comparing, for each luminosity bin, the number of counts from a trimmed version of the deeper SWIRE catalog of extragalactic sources (assumed to represent 100\% completeness by $\mathrm{c} 2 \mathrm{~d}$ standards) with the number of counts for the $\mathrm{c} 2 \mathrm{~d}$ catalogs in Serpens. This completeness estimate has been applied to all five molecular clouds observed within the c2d frame, under the assumption that the Spitzer observations for these clouds were homogeneous in terms of photometric depth. Figure 16] shows the bolometric luminosity function for Lupus V and VI before (solid line) and after (dashed line) correction for completeness and suggests that we are missing only a few lowluminosity objects with $L<0.1 L_{\odot}$, i.e. $\sim 4$ in Lupus $\mathrm{V}$ and $\sim 1$ in Lupus VI. These objects have been missed by our selection either because they are below the noise level of our GB observations or because they are located within the galaxy loci of the CM diagrams (Figure [3] 3). In conclusion, both luminosity histograms suggest a completeness better than $\sim 85 \%$ at luminosities down to $0.1 \mathrm{~L}_{\odot}$ for the YSO candidate samples in Lupus $\mathrm{V}$ and VI, which corresponds to a mass of $0.1,0.2$ and $0.35 \mathrm{M}_{\odot}$ for 1,5 and 10 Myrs old stars, respectively, according to the PMS evolutionary tracks by Baraffe et al. (1998).

Both Lupus V and VI clouds the peak of the luminosity function appears between 0.5 and $0.6 \mathrm{~L}_{\odot}$, which corresponds to a $0.6 \mathrm{M}_{\odot}$ star at an age of $1 \mathrm{Myr}$ and to a $1.3 \mathrm{M}_{\odot}$ star at an age of $5 \mathrm{Myr}$. Note that most young stars within the Lupus complex have an estimated age which falls within the 1-5 Myr interval (Comerón 2008). The bolometric luminosity histogram for the other Lupus clouds (see Figure 13 by Merín et al. 2008) suggests a typical luminosity of $0.2 L_{\odot}$, which corresponds to a $0.2 \mathrm{M}_{\odot}$ star at an age of $1 \mathrm{Myr}$ and to a $1 \mathrm{M}_{\odot}$ star at an age of 5 Myrs. Thus, YSO candidates in Lupus $\mathrm{V}$ and VI are on the average $0.4 \mathrm{M}_{\odot}$ more massive than YSOs in the other Lupus clouds. As mentioned in Sect.5, this difference may have some impact on the observed properties of disks because the disk lifetime and mass accretion rate both appear to depend on stellar mass (Brown et al. 2007; Kim et al. 2009; Merín et al. 2010; Muzerolle et al. 2010; Sicilia-Aguilar et al. 2010). Because of the large uncertainties and limitations affecting the measurement of both the disk 


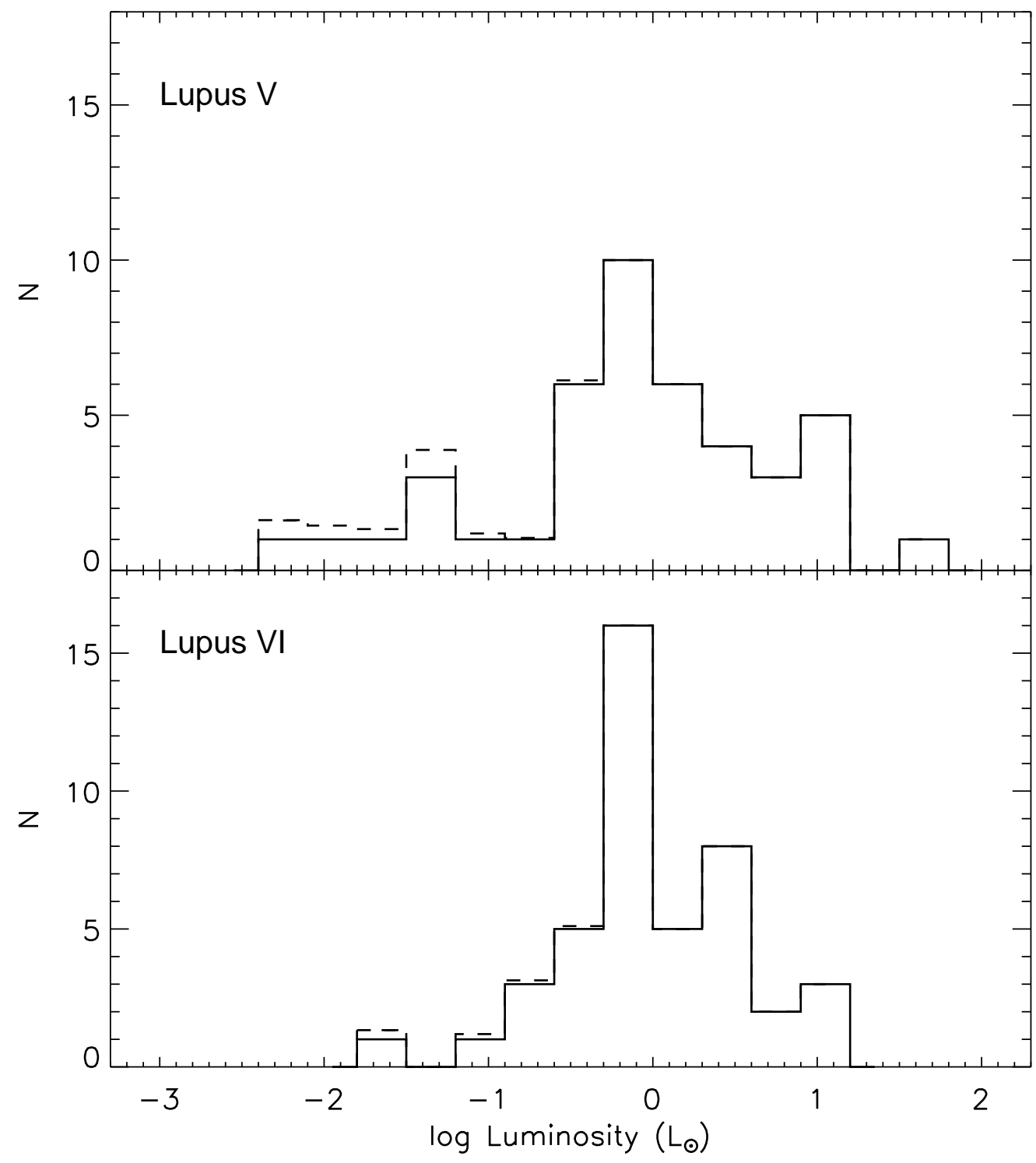

Fig. 16.- Bolometric luminosity function for the YSO candidates in Lupus V and VI before (solid line) and after correction for completeness effects (dashed line). 
lifetime and the mass accretion rate, it is still very premature to give a disk lifetime versus stellar mass calibration relation and, hence, estimate whether a $0.4 \quad \mathrm{M}_{\odot}$ difference in the characteristic stellar mass is significant. Moreover, the Lupus complex extends over about $50 \mathrm{pc}$ along the line of sight (see Sect. 3 by Comerón et al. 2009). Thus, the difference in the characteristic stellar mass we measure among the Lupus clouds might be partly due to the use of inaccurate distances for each individual cloud. However, the incorrect distance can hardly be the only cause of the greater peak in the luminosity function of Lupus $\mathrm{V}$ and VI, since bringing down the peak in luminosity (and therefore in mass) by a factor of $\sim 3$ would require these clouds to be at $\sim 70 \%$ of the assumed distance, placing them at $100 \mathrm{pc}$ or less from the Sun, which seems unlikely.

Thus, it is worth mentioning that the higher characteristic stellar mass of Lupus V and VI might be a contributing factor to the high fraction of Class III YSO candidates observed in these clouds.

\section{Summary}

We presented observations of the young stellar populations in the Lupus $\mathrm{V}$ and VI star-forming clouds at 3.6, 4.5, 5.8, 8.0, 24.0 and $70.0 \mu \mathrm{m}$ obtained with the Spitzer Space Telescope IRAC and MIPS cameras and discussed them along with optical/near-infrared data available from the literature. This study has been conducted within the frame of the Spitzer GB Survey.

The main results of this study are as follows:

- We found 43 YSO candidates in Lupus V and 45 in Lupus VI. None of them was classified as a PMS star from previous optical, near-IR and X-ray surveys;

- Most of these YSOs candidates are surrounded by thin disks as deduced from the high fraction of Class III objects ( $79 \%$ in Lupus V, $87 \%$ in Lupus VI). This frequency of Class III objects is significantly higher than measured in other clouds of the Lupus complex and in other c2d clouds;

- We found 2 potential transition objects, namely ID 3 in Lupus V and ID 19 in Lupus VI.
- The cloud density structure, the spatial distribution of the YSO candidates and the SFE in Lupus V and VI are similar to those of Lupus I and IV, but clearly differ from those of Lupus III. Lupus V and VI present both a much lower SFE and a more spatially spread population of Class III YSOs with respect to Lupus III. This suggests that the YSO population in Lupus V and VI might be a few Myrs older than the YSO population in Lupus III.

We investigate possible scenarios explaining the high frequency of Class III YSO candidates in Lupus V and VI. We prove that contamination by field stars and disk photo-evaporation due to nearby OB stars can not be responsible for the observed high fraction of class III objects, while the higher characteristic stellar mass of YSO candidates in Lupus V and VI with respect to the other Lupus clouds may be a contributing factor. Based on star formation rate and gas surface densities measurements, we conclude that star formation in Lupus V and VI ceased almost entirely a few Myrs ago, while this is not the case for other Lupus clouds. This is the most likely explanation for the observed high fraction of Class III objects. However, further observations allowing to put better constraints on the age and binary fraction of individual Lupus clouds would be necessary to definitively solve the puzzle.

We thank C. Reed for making available his allsky catalog of OB stars, A. Heiderman for providing unpublished calculations of the cloud mass in the Lupus complex and V. Roccatagliata and M. Gennaro for many useful discussions. We are also grateful to the anonymous referee for the useful revision of the paper, which helped us to improved its quality. Support for this work, part of the Spitzer Legacy Science Program, was provided by NASA through contract number 1298236 issued by the Jet Propulsion Laboratory, California Institute of Technology. Neal J. Evans acknowledges support from NSF Grant AST-0607793. 
Table 1: Lupus V and VI detection statistics.

\begin{tabular}{lll}
\hline \hline Detection criterion & \multicolumn{2}{c}{ Number of sources } \\
\hline IRAC $3.6 \mu \mathrm{m}$ with $\mathrm{S} / \mathrm{N} \geq 5$ & Lupus $\mathrm{V}$ & Lupus VI \\
IRAC $4.5 \mu \mathrm{m}$ with $\mathrm{S} / \mathrm{N} \geq 5$ & 292611 & 243185 \\
IRAC $5.8 \mu \mathrm{m}$ with $\mathrm{S} / \mathrm{N} \geq 5$ & 179095 & 168863 \\
IRAC $8.0 \mu \mathrm{m}$ with $\mathrm{S} / \mathrm{N} \geq 5$ & 33870 & 32436 \\
All 4 IRAC bands with S/N $\geq 5$ & 18366 & 17548 \\
MIPS $24 \mu \mathrm{m}$ with $\mathrm{S} / \mathrm{N} \geq 5$ & 14571 & 13632 \\
MIPS $70 \mu \mathrm{m}$ with $\mathrm{S} / \mathrm{N} \geq 5$ & 1816 & 2469 \\
All 4 IRAC bands, MIPS $24 \mu \mathrm{m}, \& \mathrm{~K}$ with $\mathrm{S} / \mathrm{N} \geq 5$ & 126 & 87 \\
\hline
\end{tabular}


Table 2: IRAC and MIPS observed fluxes for the YSO candidates in Lupus V.

\begin{tabular}{|c|c|c|c|c|c|c|c|}
\hline ID & $\begin{array}{l}\text { Name/Position } \\
\text { (SSTgbsJ) }\end{array}$ & $\begin{array}{l}3.6 \mu \mathrm{m} \\
(\mathrm{mJy})\end{array}$ & $\begin{array}{l}4.5 \mu \mathrm{m} \\
(\mathrm{mJy})\end{array}$ & $\begin{array}{l}5.8 \mu \mathrm{m} \\
(\mathrm{mJy})\end{array}$ & $\begin{array}{l}8.0 \mu \mathrm{m} \\
(\mathrm{mJy})\end{array}$ & $\begin{array}{l}24 \mu \mathrm{m} \\
(\mathrm{mJy})\end{array}$ & $\begin{array}{l}70 \mu \mathrm{m} \\
(\mathrm{mJy})\end{array}$ \\
\hline 1 & $16163197-3704563$ & $2000.00 \pm 169.00$ & $2270.00 \pm 132.00$ & $2360.00 \pm 113.00$ & $1790.00 \pm 90.80$ & $259.00 \pm 26.80$ & $81.70 \pm 10.20$ \\
\hline 2 & $16164035-3725054$ & $1860.00 \pm 109.00$ & $1500.00 \pm 80.90$ & $1330.00 \pm 64.70$ & $1010.00 \pm 48.20$ & $499.00 \pm 46.60$ & - \\
\hline 3 & $16164198-3650456$ & $1.54 \pm 0.08$ & $1.03 \pm 0.05$ & $0.72 \pm 0.05$ & $0.41 \pm 0.04$ & $12.20 \pm 1.17$ & - \\
\hline 4 & 16171811-3646307 & $4.64 \pm 0.24$ & $3.96 \pm 0.19$ & $3.67 \pm 0.18$ & $3.89 \pm 0.20$ & $5.78 \pm 0.58$ & - \\
\hline 5 & $16172474-3657333$ & $178.00 \pm 8.60$ & $167.00 \pm 8.42$ & $146.00 \pm 6.91$ & $122.00 \pm 5.91$ & $152.00 \pm 14.10$ & $468.00 \pm 49.20$ \\
\hline 6 & $16172485-3657407$ & $21.90 \pm 1.04$ & $14.30 \pm 0.70$ & $9.79 \pm 0.47$ & $5.58 \pm 0.28$ & $4.74 \pm 1.17$ & - \\
\hline 7 & $16175383-3645207$ & $2280.00 \pm 293.00$ & $3200.00 \pm 221.00$ & $3500.00 \pm 167.00$ & $2200.00 \pm 119.00$ & $237.00 \pm 22.00$ & - \\
\hline 8 & $16175958-3719083$ & $205.00 \pm 10.10$ & $120.00 \pm 6.02$ & $104.00 \pm 4.91$ & $72.40 \pm 3.52$ & $33.90 \pm 3.16$ & - \\
\hline 9 & $16180425-3710277$ & $21.20 \pm 1.03$ & $13.20 \pm 0.63$ & $9.84 \pm 0.48$ & $7.24 \pm 0.34$ & $1.58 \pm 0.25$ & - \\
\hline 10 & $16180702-3706544$ & $2460.00 \pm 144.00$ & $1780.00 \pm 123.00$ & $1590.00 \pm 78.90$ & $1090.00 \pm 52.50$ & $328.00 \pm 30.60$ & - \\
\hline 11 & $16180915-3725364$ & $248.00 \pm 12.70$ & $163.00 \pm 10.00$ & $132.00 \pm 6.49$ & $86.90 \pm 4.16$ & $21.30 \pm 1.98$ & - \\
\hline 12 & $16181727-3700597$ & $601.00 \pm 30.10$ & $345.00 \pm 19.30$ & $291.00 \pm 14.30$ & $176.00 \pm 8.45$ & $47.20 \pm 4.38$ & - \\
\hline 13 & $16183414-3715488$ & $344.00 \pm 17.50$ & $214.00 \pm 11.00$ & $193.00 \pm 9.41$ & $129.00 \pm 6.09$ & $33.60 \pm 3.13$ & - \\
\hline 14 & $16191403-3747281$ & $99.70 \pm 5.00$ & $85.60 \pm 4.20$ & $67.40 \pm 3.29$ & $59.20 \pm 2.80$ & $73.30 \pm 6.78$ & $241.00 \pm 28.30$ \\
\hline 15 & $16192683-3651238$ & $7.95 \pm 0.38$ & $6.98 \pm 0.34$ & $5.89 \pm 0.28$ & $5.24 \pm 0.25$ & $3.33 \pm 0.37$ & - \\
\hline 16 & $16194163-3746568$ & $85.60 \pm 4.32$ & $56.40 \pm 2.73$ & $47.70 \pm 2.27$ & $32.60 \pm 1.56$ & $10.20 \pm 0.96$ & - \\
\hline 17 & 16203160-3730407 & $2070.00 \pm 122.00$ & $1240.00 \pm 81.50$ & $1270.00 \pm 73.70$ & $910.00 \pm 56.50$ & $211.00 \pm 19.70$ & - \\
\hline 18 & $16205438-3742415$ & $614.00 \pm 31.90$ & $399.00 \pm 22.20$ & $324.00 \pm 16.10$ & $200.00 \pm 9.45$ & $46.80 \pm 4.34$ & - \\
\hline 19 & $16205449-3654430$ & $224.00 \pm 11.20$ & $116.00 \pm 5.69$ & $99.70 \pm 4.71$ & $64.30 \pm 3.03$ & $25.10 \pm 2.33$ & - \\
\hline 20 & $16212600-3731015$ & $757.00 \pm 40.90$ & $529.00 \pm 27.20$ & $518.00 \pm 26.00$ & $387.00 \pm 18.20$ & $161.00 \pm 14.90$ & - \\
\hline 21 & 16212991-3702213 & $189.00 \pm 9.34$ & $99.40 \pm 4.80$ & $83.70 \pm 3.92$ & $57.90 \pm 2.73$ & $56.90 \pm 5.27$ & - \\
\hline 22 & $16215125-3658489$ & $2190.00 \pm 147.00$ & $2100.00 \pm 126.00$ & $2040.00 \pm 98.90$ & $2020.00 \pm 111.00$ & $976.00 \pm 91.40$ & $83.10 \pm 16.20$ \\
\hline 23 & $16215624-3713167$ & $243.00 \pm 12.40$ & $131.00 \pm 6.58$ & $115.00 \pm 5.47$ & $73.80 \pm 3.57$ & $28.40 \pm 2.64$ & - \\
\hline 24 & $16215748-3656219$ & $81.90 \pm 4.03$ & $51.40 \pm 2.44$ & $38.30 \pm 1.81$ & $25.90 \pm 1.21$ & $5.99 \pm 0.59$ & - \\
\hline 25 & $16220163-3716369$ & $65.00 \pm 3.16$ & $42.30 \pm 2.08$ & $35.30 \pm 1.67$ & $24.80 \pm 1.20$ & $8.24 \pm 0.79$ & - \\
\hline 26 & $16221789-3658165$ & $342.00 \pm 17.30$ & $195.00 \pm 9.72$ & $168.00 \pm 8.19$ & $108.00 \pm 5.13$ & $34.40 \pm 3.20$ & - \\
\hline 27 & $16223532-3706530$ & $83.70 \pm 4.10$ & $47.30 \pm 2.29$ & $38.70 \pm 1.82$ & $24.00 \pm 1.13$ & $5.59 \pm 0.55$ & - \\
\hline 28 & $16224265-3721232$ & $361.00 \pm 18.60$ & $274.00 \pm 13.90$ & $247.00 \pm 12.50$ & $261.00 \pm 13.00$ & $198.00 \pm 18.30$ & - \\
\hline 29 & 16225309-3724374 & $298.00 \pm 14.80$ & $234.00 \pm 11.70$ & $202.00 \pm 9.70$ & $135.00 \pm 6.43$ & $22.40 \pm 2.09$ & $81.00 \pm 17.60$ \\
\hline 30 & 16230368-3729411 & $66.40 \pm 3.19$ & $41.70 \pm 2.05$ & $35.50 \pm 1.67$ & $31.20 \pm 1.51$ & $18.60 \pm 1.73$ & - \\
\hline 31 & 16232050-3741193 & $183.00 \pm 9.33$ & $165.00 \pm 7.97$ & $139.00 \pm 6.64$ & $125.00 \pm 6.00$ & $97.60 \pm 9.08$ & - \\
\hline 32 & $16232638-3752082$ & $324.00 \pm 16.50$ & $247.00 \pm 12.80$ & $233.00 \pm 11.80$ & $174.00 \pm 8.28$ & $49.60 \pm 4.61$ & - \\
\hline 33 & $16233246-3752361$ & $568.00 \pm 28.80$ & $323.00 \pm 16.60$ & $285.00 \pm 13.60$ & $192.00 \pm 9.13$ & $70.90 \pm 6.58$ & - \\
\hline 34 & $16233857-3735169$ & $149.00 \pm 7.42$ & $95.90 \pm 4.79$ & $74.60 \pm 3.53$ & $50.00 \pm 2.38$ & $14.90 \pm 1.39$ & - \\
\hline 35 & $16233865-3731388$ & $143.00 \pm 6.89$ & $75.90 \pm 3.74$ & $70.50 \pm 3.32$ & $51.60 \pm 2.44$ & $31.40 \pm 2.92$ & - \\
\hline 36 & $16234903-3727291$ & $2.10 \pm 0.10$ & $2.67 \pm 0.13$ & $3.07 \pm 0.15$ & $3.36 \pm 0.17$ & $6.46 \pm 0.67$ & - \\
\hline 37 & $16235052-3756560$ & $2420.00 \pm 175.00$ & $2130.00 \pm 98.60$ & $1790.00 \pm 88.80$ & $1240.00 \pm 63.20$ & $284.00 \pm 26.30$ & - \\
\hline 38 & $16235907-3753528$ & $2510.00 \pm 143.00$ & $1610.00 \pm 80.20$ & $1410.00 \pm 68.30$ & $950.00 \pm 45.00$ & $319.00 \pm 30.60$ & - \\
\hline 39 & 16242666-3757407 & $410.00 \pm 20.80$ & $214.00 \pm 11.00$ & $202.00 \pm 9.78$ & $129.00 \pm 6.21$ & $56.20 \pm 5.21$ & - \\
\hline 40 & $16243230-3754398$ & $855.00 \pm 43.30$ & $607.00 \pm 30.00$ & $533.00 \pm 26.70$ & $428.00 \pm 20.30$ & $170.00 \pm 15.70$ & - \\
\hline 41 & $16250690-3803214$ & $1640.00 \pm 88.20$ & $941.00 \pm 50.50$ & $820.00 \pm 39.90$ & $539.00 \pm 25.90$ & $178.00 \pm 16.50$ & - \\
\hline 42 & 16182188-3730299 & $11.20 \pm 0.91$ & $5.57 \pm 0.43$ & $4.09 \pm 0.28$ & $3.05 \pm 0.19$ & $1.73 \pm 0.31$ & $73.30 \pm 10.90$ \\
\hline 43 & $16182852-3739386$ & $7.74 \pm 0.56$ & $4.28 \pm 0.26$ & $3.05 \pm 0.19$ & $2.00 \pm 0.11$ & $1.18 \pm 0.24$ & - \\
\hline
\end{tabular}


Table 3: IRAC and MIPS observed fluxes for the YSO candidates in Lupus VI.

\begin{tabular}{|c|c|c|c|c|c|c|c|}
\hline ID & $\begin{array}{l}\text { Name/Position } \\
(\mathrm{SSTgbsJ})\end{array}$ & $\begin{array}{l}3.6 \mu \mathrm{m} \\
(\mathrm{mJy})\end{array}$ & $\begin{array}{l}4.5 \mu \mathrm{m} \\
(\mathrm{mJy})\end{array}$ & $\begin{array}{l}5.8 \mu \mathrm{m} \\
(\mathrm{mJy})\end{array}$ & $\begin{array}{l}8.0 \mu \mathrm{m} \\
(\mathrm{mJy})\end{array}$ & $\begin{array}{l}24 \mu \mathrm{m} \\
(\mathrm{mJy})\end{array}$ & $\begin{array}{l}70 \mu \mathrm{m} \\
(\mathrm{mJy})\end{array}$ \\
\hline 1 & $16200205-4137264$ & $727.00 \pm 37.20$ & $428.00 \pm 22.70$ & $360.00 \pm 17.20$ & $231.00 \pm 10.90$ & $90.00 \pm 8.38$ & - \\
\hline 2 & $16200950-4126003$ & $1400.00 \pm 71.90$ & $877.00 \pm 47.00$ & $788.00 \pm 40.20$ & $520.00 \pm 25.00$ & $161.00 \pm 14.90$ & - \\
\hline 3 & $16210869-4116025$ & $80.50 \pm 3.96$ & $50.40 \pm 2.42$ & $40.00 \pm 1.88$ & $28.10 \pm 1.32$ & $6.71 \pm 0.65$ & - \\
\hline 4 & $16211125-4120172$ & $47.60 \pm 2.50$ & $28.90 \pm 1.38$ & $23.80 \pm 1.14$ & $16.20 \pm 0.77$ & $6.17 \pm 0.62$ & - \\
\hline 5 & $16213597-4121525$ & $825.00 \pm 43.40$ & $455.00 \pm 23.30$ & $381.00 \pm 18.60$ & $234.00 \pm 12.30$ & $60.30 \pm 5.58$ & - \\
\hline 6 & $16213962-4109135$ & $141.00 \pm 7.11$ & $96.30 \pm 4.76$ & $76.00 \pm 3.59$ & $52.10 \pm 2.45$ & $12.80 \pm 1.20$ & - \\
\hline 7 & $16214077-4122218$ & $158.00 \pm 7.92$ & $86.30 \pm 4.38$ & $73.60 \pm 3.45$ & $48.60 \pm 2.31$ & $19.20 \pm 1.79$ & - \\
\hline 8 & $16214973-4107017$ & $192.00 \pm 9.39$ & $134.00 \pm 6.60$ & $108.00 \pm 5.09$ & $74.80 \pm 3.59$ & $24.00 \pm 2.23$ & - \\
\hline 9 & $16215797-4131099$ & $167.00 \pm 8.46$ & $103.00 \pm 5.23$ & $86.40 \pm 4.09$ & $55.70 \pm 2.70$ & $12.20 \pm 1.14$ & - \\
\hline 10 & $16222724-4100410$ & $198.00 \pm 9.83$ & $114.00 \pm 5.62$ & $91.30 \pm 4.28$ & $60.20 \pm 2.84$ & $18.60 \pm 1.73$ & - \\
\hline 11 & $16222844-4113424$ & $252.00 \pm 12.90$ & $149.00 \pm 7.15$ & $125.00 \pm 5.95$ & $80.60 \pm 3.82$ & $24.60 \pm 2.28$ & - \\
\hline 12 & $16222966-4123457$ & $1840.00 \pm 206.00$ & $2670.00 \pm 168.00$ & $3480.00 \pm 220.00$ & $2820.00 \pm 136.00$ & $1060.00 \pm 101.00$ & - \\
\hline 13 & $16223341-4103179$ & $822.00 \pm 45.10$ & $495.00 \pm 25.00$ & $426.00 \pm 20.40$ & $287.00 \pm 13.60$ & $121.00 \pm 11.20$ & - \\
\hline 14 & $16230307-4021119$ & $651.00 \pm 40.60$ & $379.00 \pm 20.10$ & $377.00 \pm 20.10$ & $231.00 \pm 11.10$ & $82.70 \pm 7.68$ & - \\
\hline 15 & $16231101-4117103$ & $167.00 \pm 8.61$ & $115.00 \pm 5.88$ & $92.10 \pm 4.35$ & $61.60 \pm 2.92$ & $17.90 \pm 1.67$ & - \\
\hline 16 & $16231476-4017151$ & $220.00 \pm 11.70$ & $136.00 \pm 6.71$ & $115.00 \pm 5.56$ & $81.50 \pm 3.89$ & $28.60 \pm 2.67$ & - \\
\hline 17 & $16231598-4104009$ & $1800.00 \pm 126.00$ & $1310.00 \pm 70.60$ & $1120.00 \pm 55.30$ & $736.00 \pm 35.40$ & $241.00 \pm 22.50$ & - \\
\hline 18 & $16231844-4019439$ & $1490.00 \pm 147.00$ & $2080.00 \pm 107.00$ & $1660.00 \pm 87.60$ & $1170.00 \pm 55.30$ & $210.00 \pm 19.50$ & - \\
\hline 19 & $16232809-4015368$ & $37.70 \pm 1.87$ & $22.80 \pm 1.09$ & $16.70 \pm 0.87$ & $9.83 \pm 0.47$ & $31.10 \pm 2.90$ & $771.00 \pm 81.10$ \\
\hline 20 & $16233735-4014490$ & $136.00 \pm 6.91$ & $70.80 \pm 3.64$ & $60.50 \pm 2.93$ & $38.50 \pm 1.82$ & $11.40 \pm 1.07$ & - \\
\hline 21 & $16234486-4107493$ & $595.00 \pm 31.30$ & $470.00 \pm 27.30$ & $407.00 \pm 20.20$ & $302.00 \pm 15.40$ & $98.80 \pm 9.16$ & - \\
\hline 22 & $16234903-4026176$ & $164.00 \pm 8.15$ & $113.00 \pm 5.66$ & $97.20 \pm 4.59$ & $77.90 \pm 3.70$ & $25.20 \pm 2.34$ & - \\
\hline 23 & $16235138-4010324$ & $262.00 \pm 12.80$ & $157.00 \pm 7.80$ & $136.00 \pm 6.44$ & $90.40 \pm 4.26$ & $20.20 \pm 1.87$ & - \\
\hline 24 & $16242396-3952100$ & $1020.00 \pm 51.30$ & $552.00 \pm 31.80$ & $527.00 \pm 25.00$ & $332.00 \pm 15.90$ & $91.80 \pm 8.56$ & $45.10 \pm 8.36$ \\
\hline 25 & $16242615-4011026$ & $232.00 \pm 11.90$ & $148.00 \pm 7.77$ & $123.00 \pm 5.85$ & $81.80 \pm 3.96$ & $20.00 \pm 1.85$ & - \\
\hline 26 & $16244169-4004196$ & $99.10 \pm 5.10$ & $59.40 \pm 2.92$ & $45.70 \pm 2.20$ & $29.90 \pm 1.41$ & $8.05 \pm 0.76$ & - \\
\hline 27 & $16244645-3956150$ & $56.10 \pm 2.72$ & $51.80 \pm 2.60$ & $47.60 \pm 2.23$ & $37.40 \pm 1.79$ & $45.00 \pm 4.16$ & - \\
\hline 28 & $16245178-3956326$ & $42.90 \pm 2.13$ & $60.30 \pm 3.07$ & $86.00 \pm 4.06$ & $104.00 \pm 5.18$ & $211.00 \pm 19.50$ & $110.00 \pm 15.60$ \\
\hline 29 & $16245564-3949147$ & $109.00 \pm 6.76$ & $99.40 \pm 4.81$ & $73.00 \pm 3.59$ & $52.30 \pm 2.46$ & $12.80 \pm 1.20$ & - \\
\hline 30 & $16245590-4011282$ & $1430.00 \pm 101.00$ & $949.00 \pm 54.00$ & $954.00 \pm 50.50$ & $674.00 \pm 33.50$ & $178.00 \pm 16.50$ & - \\
\hline 31 & $16245681-4008238$ & $250.00 \pm 15.90$ & $214.00 \pm 10.90$ & $184.00 \pm 8.90$ & $119.00 \pm 5.93$ & $24.40 \pm 2.27$ & - \\
\hline 32 & $16255246-4018484$ & $123.00 \pm 9.05$ & $79.60 \pm 4.95$ & $73.80 \pm 3.74$ & $46.80 \pm 2.37$ & $12.80 \pm 1.20$ & - \\
\hline 33 & $16255837-4009581$ & $777.00 \pm 42.80$ & $423.00 \pm 26.50$ & $406.00 \pm 20.50$ & $263.00 \pm 13.20$ & $105.00 \pm 9.73$ & - \\
\hline 34 & $16260853-4017441$ & $459.00 \pm 30.80$ & $241.00 \pm 14.90$ & $246.00 \pm 14.30$ & $153.00 \pm 7.66$ & $33.20 \pm 3.10$ & - \\
\hline 35 & 16261339-3949542 & $215.00 \pm 12.70$ & $160.00 \pm 8.55$ & $134.00 \pm 6.98$ & $101.00 \pm 5.01$ & $37.00 \pm 3.44$ & - \\
\hline 36 & $16261365-4012186$ & $636.00 \pm 36.00$ & $286.00 \pm 21.70$ & $317.00 \pm 16.70$ & $214.00 \pm 11.20$ & $52.50 \pm 4.87$ & - \\
\hline 37 & $16262551-3944472$ & $47.00 \pm 2.64$ & $32.00 \pm 1.65$ & $25.20 \pm 1.29$ & $29.40 \pm 1.43$ & $15.30 \pm 1.42$ & - \\
\hline 38 & $16263114-3946153$ & $2.76 \pm 0.16$ & $1.70 \pm 0.09$ & $1.31 \pm 0.08$ & $0.85 \pm 0.05$ & $0.84 \pm 0.22$ & - \\
\hline 39 & $16263155-4004557$ & $597.00 \pm 32.80$ & $375.00 \pm 21.70$ & $313.00 \pm 17.30$ & $209.00 \pm 10.30$ & $50.60 \pm 4.69$ & - \\
\hline 40 & $16263925-4015272$ & $393.00 \pm 24.80$ & $263.00 \pm 16.10$ & $310.00 \pm 16.30$ & $212.00 \pm 10.60$ & $115.00 \pm 10.60$ & - \\
\hline 41 & $16265364-3954594$ & $283.00 \pm 17.30$ & $223.00 \pm 12.50$ & $200.00 \pm 10.50$ & $136.00 \pm 6.73$ & $21.00 \pm 1.95$ & - \\
\hline 42 & $16270309-3944139$ & $293.00 \pm 20.00$ & $255.00 \pm 12.90$ & $199.00 \pm 11.70$ & $154.00 \pm 7.27$ & $32.90 \pm 3.06$ & - \\
\hline 43 & $16273175-3956013$ & $70.70 \pm 3.66$ & $41.20 \pm 2.15$ & $35.50 \pm 1.68$ & $22.40 \pm 1.10$ & $5.59 \pm 0.56$ & - \\
\hline 44 & $16275054-3948100$ & $471.00 \pm 27.70$ & $281.00 \pm 13.80$ & $239.00 \pm 11.40$ & $159.00 \pm 7.51$ & $57.40 \pm 5.31$ & - \\
\hline 45 & $16282647-3925428$ & $126.00 \pm 6.61$ & $139.00 \pm 6.88$ & $188.00 \pm 9.34$ & $272.00 \pm 13.00$ & $175.00 \pm 16.30$ & - \\
\hline
\end{tabular}


Table 4: NOMAD, DENIS and 2MASS observed magnitudes for the YSO candidates in Lupus V.

\begin{tabular}{|c|c|c|c|c|c|c|c|c|}
\hline ID & $\begin{array}{l}\text { Name/Position } \\
\text { (SSTgbsJ) }\end{array}$ & $\mathrm{B}^{\dagger}$ & $\mathrm{V}^{\dagger}$ & $\mathrm{R}^{\dagger}$ & I & $\mathrm{J}$ & $\mathrm{H}$ & $\mathrm{K}_{S}$ \\
\hline 1 & $16163197-3704563$ & 17.85 & 15.31 & - & - & $7.06 \pm 0.02$ & $5.72 \pm 0.03$ & $5.10 \pm 0.02$ \\
\hline 2 & $16164035-3725054$ & 18.49 & - & - & - & $8.46 \pm 0.02$ & $7.28 \pm 0.04$ & $6.43 \pm 0.03$ \\
\hline 3 & $16164198-3650456$ & 19.30 & - & 17.47 & - & $14.41 \pm 0.03$ & $13.58 \pm 0.03$ & $13.29 \pm 0.03$ \\
\hline 4 & 16171811-3646307 & 19.89 & - & 18.10 & $16.05 \pm 0.06$ & $13.66 \pm 0.02$ & $12.99 \pm 0.02$ & $12.66 \pm 0.03$ \\
\hline 5 & $16172474-3657333$ & 17.25 & 16.44 & - & $13.24 \pm 0$ & $10.56 \pm 0.04$ & $9.61 \pm 0.04$ & $9.09 \pm 0.03$ \\
\hline 6 & $16172485-3657407$ & 16.96 & 15.62 & 14.29 & $13.02 \pm 0.03$ & $11.47 \pm 0.03$ & $10.83 \pm 0.03$ & $10.59 \pm 0.02$ \\
\hline 7 & $16175383-3645207$ & 17.61 & - & 9.85 & $8.59 \pm 0.04$ & $5.59 \pm 0.02$ & $4.48 \pm 0.23$ & $4.11 \pm 0.23$ \\
\hline 8 & $16175958-3719083$ & - & - & 18.05 & $14.43 \pm 0.05$ & $10.10 \pm 0.02$ & $8.85 \pm 0.02$ & $8.18 \pm 0.03$ \\
\hline 9 & $16180425-3710277$ & 18.38 & 16.82 & 14.86 & $14.18 \pm 0.04$ & $12.05 \pm 0.03$ & $10.92 \pm 0.04$ & $10.50 \pm 0.02$ \\
\hline 10 & $16180702-3706544$ & - & 17.74 & - & $11.39 \pm 0.02$ & $7.34 \pm 0.03$ & $6.04 \pm 0.03$ & $5.33 \pm 0.02$ \\
\hline 11 & $16180915-3725364$ & - & 17.32 & 15.91 & $12.71 \pm 0.02$ & $9.69 \pm 0.02$ & $8.40 \pm 0.05$ & $7.94 \pm 0.03$ \\
\hline 12 & $16181727-3700597$ & 18.93 & 17.18 & 15.68 & $12.10 \pm 0.02$ & $8.89 \pm 0.02$ & $7.61 \pm 0.03$ & $7.00 \pm 0.02$ \\
\hline 13 & $16183414-3715488$ & 19.44 & 17.74 & - & $13.62 \pm 0.04$ & $9.64 \pm 0.02$ & $8.30 \pm 0.06$ & $7.72 \pm 0.05$ \\
\hline 14 & $16191403-3747281$ & 15.72 & 14.44 & 13.36 & $13.13 \pm 0.03$ & $11.69 \pm 0.02$ & $10.63 \pm 0.02$ & $9.92 \pm 0.02$ \\
\hline 15 & $16192683-3651238$ & 17.95 & - & 16.93 & 15.41 & $13.13 \pm 0.03$ & $12.56 \pm 0.03$ & $12.16 \pm 0.03$ \\
\hline 16 & $16194163-3746568$ & 19.45 & - & - & 14.27 & $10.98 \pm 0.02$ & $9.77 \pm 0.02$ & $9.25 \pm 0.02$ \\
\hline 17 & $16203160-3730407$ & - & 17.32 & - & $12.03 \pm$ & $7.64 \pm 0.02$ & $6.42 \pm 0.02$ & $5.74 \pm 0.02$ \\
\hline 18 & $16205438-3742415$ & 18.27 & - & 15.23 & 12. & $8.7^{\circ}$ & $7.53 \pm 0.03$ & $6.98 \pm 0.02$ \\
\hline 19 & $16205449-3654430$ & 18.47 & - & - & $13.11 \pm 1.00$ & 9.82 & $8.56 \pm 0$ & $=0.02$ \\
\hline 20 & $16212600-3$ & - & - & 16. & - & 02 & $8.47 \pm 0.02$ & $7.54 \pm 0.06$ \\
\hline 21 & 16212991-3702213 & 18.38 & 16.07 & 14.92 & 12. & 9.94 & $8.78 \pm 0.02$ & $8.26 \pm 0.02$ \\
\hline 22 & $16215125-3658489$ & 18.35 & 16.50 & - & 11.5 & $7.08 \pm 0.02$ & $5.90 \pm 0.03$ & $5.19 \pm 0.02$ \\
\hline 23 & $16215624-3713167$ & 19.19 & - & - & $13.64 \pm$ & $9.78 \pm 0.02$ & $8.55 \pm 0.02$ & $7.97 \pm 0.02$ \\
\hline 24 & $16215748-3656219$ & 18.38 & 16.37 & 14.95 & $13.24 \pm 0.03$ & $10.75 \pm 0.02$ & $9.55 \pm 0.02$ & $9.13 \pm 0.02$ \\
\hline 25 & $16220163-3716369$ & 18.42 & 17.28 & 16.20 & $13.61 \pm 0.03$ & $11.16 \pm 0.02$ & $9.96 \pm 0.02$ & $9.46 \pm 0.02$ \\
\hline 26 & $16221789-3658165$ & 18.73 & - & - & $13.07 \pm 0.03$ & $9.43 \pm 0.02$ & $8.23 \pm 0.02$ & $7.64 \pm 0.02$ \\
\hline 27 & $16223532-3706530$ & 19.33 & - & 17.17 & $14.18 \pm 0.03$ & $10.90 \pm 0.02$ & $9.63 \pm 0.02$ & $9.10 \pm 0.02$ \\
\hline 28 & $16224265-3721232$ & 18.82 & - & 16.08 & $13.21 \pm 0.03$ & $9.77 \pm 0.02$ & $8.43 \pm 0.02$ & $7.82 \pm 0.03$ \\
\hline 29 & $16225309-3724374$ & 18.33 & 16.44 & - & - & $9.96 \pm 0.02$ & $8.73 \pm 0.02$ & $8.13 \pm 0.03$ \\
\hline 30 & $16230368-3729411$ & 17.88 & 17.41 & 16.02 & $13.98 \pm 0.03$ & \pm 0.02 & $9.84 \pm 0.02$ & $9.36 \pm 0.02$ \\
\hline 31 & $16232050-3741193$ & - & - & 18.09 & $14.09 \pm 0.05$ & $10.84 \pm 0.02$ & $9.67 \pm 0.02$ & $8.9 \pm 0.02$ \\
\hline 32 & $16232638-3752082$ & - & - & 17.31 & - & $10.31 \pm 0.02$ & $9.10 \pm 0.02$ & $8.37 \pm 0.02$ \\
\hline 33 & $16233246-3752361$ & 18.35 & 16.67 & - & $11.88 \pm 0.04$ & $8.82 \pm 0.02$ & $7.55 \pm 0.02$ & $7.00 \pm 0.02$ \\
\hline 34 & $16233857-3735169$ & 18.10 & 17.15 & - & 12.8 & 10.1 & $8.96 \pm 0.02$ & $8.50 \pm 0.02$ \\
\hline 35 & -3731388 & 18.58 & 17.74 & 15.80 & $13.10 \pm 0.05$ & $10.25 \pm 0.02$ & $9.08 \pm 0.02$ & $8.55 \pm 0.02$ \\
\hline 36 & $16234903-3727291$ & - & - & - & - & - & - & $15.08 \pm 0.16$ \\
\hline 37 & $16235052-3756560$ & 18.31 & 16.11 & 14.4 & $10.94 \pm$ & $6.97=$ & $5.66 \pm 0.03$ & $5.08 \pm 0.01$ \\
\hline 38 & $16235907-3753528$ & 18.38 & 15.99 & 14.28 & $10.74 \pm 0.06$ & $7.14 \pm 0$ & $5.87 \pm 0.05$ & $5.28 \pm 0.03$ \\
\hline 39 & $16242666-3757407$ & 19.57 & - & - & $13.52 \pm 0.03$ & $9.27 \pm 0.02$ & $7.97 \pm 0.05$ & $7.35 \pm 0.02$ \\
\hline 40 & $16243230-3754398$ & 17.49 & - & - & $11.76 \pm 0.02$ & $8.43 \pm 0.03$ & $7.20 \pm 0.03$ & $6.70 \pm 0.02$ \\
\hline 41 & $16250690-3803214$ & 18.38 & 16.69 & 14.99 & $11.49 \pm 0.02$ & $7.69 \pm 0.02$ & $6.44 \pm 0.02$ & $5.87 \pm 0.02$ \\
\hline 42 & 16182188-3730299 & 17.86 & - & - & $14.54 \pm 0.05$ & $12.79 \pm 0.10$ & $11.73 \pm 0.11$ & $11.31 \pm 0.09$ \\
\hline 43 & $16182852-3739386$ & 17.40 & - & - & $15.36 \pm 0.07$ & $13.32 \pm 0.07$ & $12.30 \pm 0.09$ & $11.77 \pm 0.06$ \\
\hline
\end{tabular}

$\dagger$ Photometric uncertainties of the NOMAD magnitudes are of the order of 1-2\% (Zacharias et al. 2005). 
Table 5: NOMAD, DENIS and 2MASS observed magnitudes for the YSO candidates in Lupus VI.

\begin{tabular}{|c|c|c|c|c|c|c|c|c|}
\hline ID & $\begin{array}{l}\text { Name/Position } \\
\text { (SSTgbsJ) }\end{array}$ & $\mathrm{B}^{\dagger}$ & $\mathrm{V}^{\dagger}$ & $\mathrm{R}^{\dagger}$ & $\mathrm{I}$ & $\mathrm{J}$ & $\overline{\mathrm{H}}$ & $\mathrm{K}_{S}$ \\
\hline 1 & $16200205-4137264$ & 17.31 & - & - & $12.06 \pm 0.02$ & $8.53 \pm 0.03$ & $7.29 \pm 0.03$ & $6.73 \pm 0.03$ \\
\hline 2 & $16200950-4126003$ & 17.56 & - & - & $12.46 \pm 0.02$ & $8.29 \pm 0.02$ & $6.91 \pm 0.03$ & $6.28 \pm 0.01$ \\
\hline 3 & $16210869-4116025$ & 17.33 & - & 16.29 & $13.72 \pm 1.00$ & $10.83 \pm 0.02$ & $9.62 \pm 0.02$ & $9.14 \pm 0.02$ \\
\hline 4 & $16211125-4120172$ & - & - & 18.71 & $15.40 \pm 1.00$ & $11.80 \pm 0.02$ & $10.48 \pm 0.02$ & $9.94 \pm 0.02$ \\
\hline 5 & $16213597-4121525$ & 17.74 & 16.26 & 14.94 & $11.86 \pm 0.03$ & $8.35 \pm 0.02$ & $7.12 \pm 0.02$ & $6.55 \pm 0.02$ \\
\hline 6 & $16213962-4109135$ & 18.84 & - & 16.17 & $13.31 \pm 0.03$ & $10.29 \pm 0.02$ & $9.05 \pm 0.02$ & $8.58 \pm 0.02$ \\
\hline 7 & $16214077-4122218$ & 17.89 & - & 15.98 & $13.67 \pm 0.03$ & $10.26 \pm 0.02$ & $9.03 \pm 0.02$ & $8.51 \pm 0.02$ \\
\hline 8 & $16214973-4107017$ & 18.03 & - & 15.35 & $13.05 \pm 0.03$ & $10.31 \pm 0.02$ & $9.12 \pm 0.02$ & $8.50 \pm 0.02$ \\
\hline 9 & $16215797-4131099$ & 17.27 & 16.98 & 15.22 & $12.77 \pm 0.03$ & $10.08 \pm 0.02$ & $8.80 \pm 0.02$ & $8.32 \pm 0.02$ \\
\hline 10 & $16222724-4100410$ & 18.00 & - & 16.41 & $13.48 \pm 0.03$ & $10.03 \pm 0.02$ & $8.80 \pm 0.02$ & $8.24 \pm 0.02$ \\
\hline 11 & $16222844-4113424$ & - & - & 16.74 & $14.10 \pm 0.03$ & $9.98 \pm 0.02$ & $8.54 \pm 0.03$ & $7.91 \pm 0.02$ \\
\hline 12 & $16222966-4123457$ & - & - & - & $11.81 \pm 0.02$ & $7.18 \pm 0.02$ & $5.92 \pm 0.03$ & $5.07 \pm 0.02$ \\
\hline 13 & $16223341-4103179$ & 18.25 & - & - & $13.19 \pm 0.03$ & $8.61 \pm 0.03$ & $7.22 \pm 0.02$ & $6.62 \pm 0.03$ \\
\hline 14 & $16230307-4021119$ & 17.93 & - & - & $13.06 \pm 0.02$ & $9.32 \pm 0.02$ & $8.04 \pm 0.03$ & $7.38 \pm 0.03$ \\
\hline 15 & $16231101-4117103$ & 18.45 & - & 17.19 & $13.86 \pm 0.03$ & $10.36 \pm 0.03$ & $8.97 \pm 0.02$ & $8.37 \pm 0.02$ \\
\hline 16 & $16231476-4017151$ & 18.47 & - & 16.96 & $13.71 \pm 0.03$ & $9.94 \pm 0.02$ & $8.65 \pm 0.02$ & $8.03 \pm 0.02$ \\
\hline 17 & $16231598-4104009$ & 16.00 & 16.14 & 13.11 & $11.46 \pm 0.03$ & $7.27 \pm 0.02$ & $5.98 \pm 0.02$ & $5.34 \pm 0.02$ \\
\hline 18 & $16231844-4019439$ & 15.48 & 13.40 & 12.35 & $10.56 \pm 0.06$ & $6.98 \pm 0.02$ & $5.76 \pm 0.03$ & $5.25 \pm 0.02$ \\
\hline 19 & $16232809-4015368$ & - & 17.22 & - & $14.81 \pm 0.06$ & $11.82 \pm 0.03$ & $10.50 \pm 0.03$ & $10.05 \pm 0.03$ \\
\hline 20 & $16233735-4014490$ & - & - & 18.69 & $15.15 \pm 0.06$ & $10.75 \pm 0.03$ & $9.38 \pm 0.02$ & $8.73 \pm 0.02$ \\
\hline 21 & $16234486-4107493$ & - & - & - & $12.86 \pm 0.05$ & $9.83 \pm 0.02$ & $8.55 \pm 0.04$ & $7.68 \pm 0.03$ \\
\hline 22 & $16234903-4026176$ & 18.07 & - & 17.12 & $14.15 \pm 0.05$ & $10.55 \pm 0.02$ & $9.23 \pm 0.02$ & $8.64 \pm 0.02$ \\
\hline 23 & $16235138-4010324$ & 18.07 & - & 15.96 & $13.77 \pm 0.05$ & $9.84 \pm 0.02$ & $8.63 \pm 0.05$ & $8.05 \pm 0.04$ \\
\hline 24 & $16242396-3952100$ & 17.15 & - & - & $11.99 \pm 0.02$ & $8.41 \pm 0.02$ & $7.15 \pm 0.03$ & $6.56 \pm 0.02$ \\
\hline 25 & $16242615-4011026$ & - & 17.20 & - & $13.44 \pm 0.03$ & $10.16 \pm 0.02$ & $8.91 \pm 0.02$ & $8.29 \pm 0.04$ \\
\hline 26 & $16244169-4004196$ & 17.88 & 17.74 & 16.81 & $13.85 \pm 0.03$ & $10.84 \pm 0.02$ & $9.52 \pm 0.02$ & $8.97 \pm 0.02$ \\
\hline 27 & $16244645-3956150$ & - & - & - & - & $13.90 \pm 0.03$ & $11.84 \pm 0.02$ & $10.65 \pm 0.02$ \\
\hline 28 & $16245178-3956326$ & - & - & - & - & $16.20 \pm 0.12$ & $14.24 \pm 0.06$ & $12.69 \pm 0.03$ \\
\hline 29 & 16245564-3949147 & 17.27 & 16.91 & 15.93 & $13.08 \pm 0.03$ & $10.42 \pm 0.03$ & $9.30 \pm 0.02$ & $8.72 \pm 0.02$ \\
\hline 30 & $16245590-4011282$ & - & - & 15.15 & $12.07 \pm 0.02$ & $7.95 \pm 0.02$ & $6.72 \pm 0.06$ & $6.00 \pm 0.02$ \\
\hline 31 & $16245681-4008238$ & 17.39 & - & 15.31 & $12.40 \pm 0.02$ & $9.51 \pm 0.02$ & $8.26 \pm 0.05$ & $7.71 \pm 0.03$ \\
\hline 32 & $16255246-4018484$ & 18.14 & - & 17.39 & $13.75 \pm 0.03$ & $10.28 \pm 0.02$ & $8.98 \pm 0.02$ & $8.39 \pm 0.03$ \\
\hline 33 & $16255837-4009581$ & 18.53 & - & - & $13.19 \pm 0.02$ & $8.72 \pm 0.02$ & $7.33 \pm 0.05$ & $6.66 \pm 0.03$ \\
\hline 34 & $16260853-4017441$ & 16.86 & 16.68 & 15.28 & $12.12 \pm 0.02$ & $8.97 \pm 0.02$ & $7.67 \pm 0.05$ & $7.08 \pm 0.02$ \\
\hline 35 & 16261339-3949542 & 17.74 & - & 14.83 & $13.30 \pm 0.02$ & $9.94 \pm 0.02$ & $8.83 \pm 0.04$ & $8.18 \pm 0.04$ \\
\hline 36 & $16261365-4012186$ & 17.95 & 17.11 & 15.81 & $12.57 \pm 0.02$ & $8.89 \pm 0.03$ & $7.46 \pm 0.05$ & $6.80 \pm 0.02$ \\
\hline 37 & $16262551-3944472$ & 17.15 & - & 15.44 & $13.71 \pm 0.03$ & $11.31 \pm 0.02$ & $10.10 \pm 0.02$ & $9.66 \pm 0.02$ \\
\hline 38 & 16263114-3946153 & - & - & - & $15.43 \pm 0.05$ & $13.78 \pm 0.06$ & - & - \\
\hline 39 & $16263155-4004557$ & 18.00 & - & 15.84 & $12.63 \pm 0.03$ & $9.05 \pm 0.03$ & $7.65 \pm 0.04$ & $7.03 \pm 0.02$ \\
\hline 40 & $16263925-4015272$ & - & - & - & $17.10 \pm 0.12$ & $11.13 \pm 0.02$ & $9.42 \pm 0.02$ & $8.41 \pm 0.02$ \\
\hline 41 & 16265364-3954594 & 18.08 & 16.56 & - & $12.25 \pm 0.02$ & $9.94 \pm 0.02$ & $8.72 \pm 0.02$ & $8.09 \pm 0.02$ \\
\hline 42 & 16270309-3944139 & - & - & - & $12.57 \pm 0.03$ & $10.01 \pm 0.02$ & $8.80 \pm 0.02$ & $8.11 \pm 0.02$ \\
\hline 43 & $16273175-3956013$ & 18.35 & - & 16.83 & $14.44 \pm 0.03$ & $11.3 \pm 0.02$ & $9.99 \pm 0.02$ & $9.44 \pm 0.02$ \\
\hline 44 & $16275054-3948100$ & 17.84 & - & 16.20 & $12.78 \pm 0.03$ & $9.26 \pm 0.02$ & $7.97 \pm 0.05$ & $7.39 \pm 0.02$ \\
\hline 45 & $16282647-3925428$ & 16.74 & - & 14.25 & - & $11.37 \pm 0.02$ & $10.26 \pm 0.02$ & $9.80 \pm 0.02$ \\
\hline
\end{tabular}

${ }^{\dagger}$ Photometric uncertainties of the NOMAD magnitudes are of the order of 1-2\% (Zacharias et al. 2005). 
Table 6: SED slopes and Lada class for the YSO candidates in Lupus V.

\begin{tabular}{|c|c|c|c|c|c|}
\hline$\overline{\text { ID }}$ & Name/Position & $\alpha_{K-24}$ & $\alpha_{3.6-5.8}$ & $\alpha_{8}-24$ & $\overline{\text { Lada class }}$ \\
\hline 1 & $16163197-3704563$ & -2.06 & -0.66 & -2.76 & III \\
\hline 2 & $16164035-3725054$ & -1.62 & -1.70 & -1.64 & III \\
\hline $3^{*}$ & 16164198-3650456 & -0.55 & -2.59 & 2.10 & II \\
\hline 4 & $16171811-3646307$ & -1.03 & -1.49 & -0.64 & II \\
\hline 5 & $16172474-3657333$ & -1.15 & -1.42 & -0.80 & II \\
\hline 6 & $16172485-3657407$ & -2.43 & -2.68 & -1.15 & III \\
\hline 7 & $16175383-3645207$ & -2.47 & -0.11 & -3.03 & III \\
\hline 8 & $16175958-3719083$ & -2.02 & -2.40 & -1.69 & III \\
\hline 9 & $16180425-3710277$ & -2.35 & -2.60 & -2.39 & III \\
\hline 10 & $16180702-3706544$ & -2.08 & -1.90 & -2.09 & III \\
\hline 11 & $16180915-3725364$ & -2.27 & -2.31 & -2.28 & III \\
\hline 12 & $16181727-3700597$ & -2.34 & -2.50 & -2.20 & III \\
\hline 13 & $16183414-3715488$ & -2.16 & -2.19 & -2.22 & III \\
\hline 14 & $16191403-3747281$ & -1.21 & -1.82 & -0.81 & II \\
\hline 15 & $16192683-3651238$ & -1.46 & -1.63 & -1.41 & II \\
\hline 16 & $16194163-3746568$ & -2.09 & -2.21 & -2.06 & III \\
\hline 17 & $16203160-3730407$ & -2.11 & -2.00 & -2.33 & III \\
\hline 18 & $16205438-3742415$ & -2.32 & -2.33 & -2.32 & III \\
\hline 19 & $16205449-3654430$ & -2.22 & -2.67 & -1.86 & III \\
\hline 20 & $16212600-3731015$ & -1.69 & -1.78 & -1.80 & III \\
\hline 21 & $16212991-3702213$ & -1.86 & -2.68 & -1.02 & III \\
\hline 22 & $16215125-3658489$ & -1.54 & -1.15 & -1.66 & II \\
\hline 23 & $16215624-3713167$ & -2.18 & -2.54 & -1.87 & III \\
\hline 24 & $16215748-3656219$ & -2.36 & -2.58 & -2.33 & III \\
\hline 25 & $16220163-3716369$ & -2.09 & -2.27 & -2.00 & III \\
\hline 26 & $16221789-3658165$ & -2.22 & -2.47 & -2.04 & III \\
\hline 27 & $16223532-3706530$ & -2.41 & -2.60 & -2.33 & III \\
\hline 28 & $16224265-3721232$ & -1.35 & -1.79 & -1.25 & II \\
\hline 29 & $16225309-3724374$ & -2.16 & -1.81 & -2.63 & III \\
\hline 30 & $16230368-3729411$ & -1.76 & -2.30 & -1.47 & III \\
\hline 31 & $16232050-3741193$ & -1.34 & -1.58 & -1.23 & II \\
\hline 32 & $16232638-3752082$ & -1.80 & -1.68 & -2.14 & III \\
\hline 33 & $16233246-3752361$ & -2.14 & -2.42 & -1.91 & III \\
\hline 34 & $16233857-3735169$ & -2.23 & -2.44 & -2.10 & III \\
\hline 35 & $16233865-3731388$ & -1.91 & -2.46 & -1.45 & III \\
\hline 36 & $16234903-3727291$ & -0.35 & -0.21 & -0.40 & II \\
\hline 37 & $16235052-3756560$ & -2.17 & -1.63 & -2.34 & III \\
\hline 38 & $16235907-3753528$ & -2.12 & -2.19 & -1.99 & III \\
\hline 39 & $16242666-3757407$ & -2.12 & -2.46 & -1.76 & III \\
\hline 40 & $16243230-3754398$ & -1.85 & -1.98 & -1.84 & III \\
\hline 41 & $16250690-3803214$ & -2.19 & -2.43 & -2.01 & III \\
\hline 42 & 16182188-3730299 & -2.22 & -3.09 & -1.52 & III \\
\hline 43 & $16182852-3739386$ & -2.32 & -2.94 & -1.48 & III \\
\hline
\end{tabular}

*Transition object candidate. 
Table 7: SED slopes and Lada class for the YSO candidates in Lupus VI.

\begin{tabular}{|c|c|c|c|c|c|}
\hline$\overline{\text { ID }}$ & Name/Position & $\alpha_{K-24}$ & $\alpha_{3.6-5.8}$ & $\alpha_{8}-24$ & Lada class \\
\hline 1 & $16200205-4137264$ & -2.17 & -2.46 & -1.86 & III \\
\hline 2 & $16200950-4126003$ & -2.09 & -2.19 & -2.07 & III \\
\hline 3 & $16210869-4116025$ & -2.27 & -2.45 & -2.30 & III \\
\hline 4 & $16211125-4120172$ & -2.09 & -2.44 & -1.88 & III \\
\hline 5 & $16213597-4121525$ & -2.38 & -2.60 & -2.23 & III \\
\hline 6 & $16213962-4109135$ & -2.23 & -2.29 & -2.28 & III \\
\hline 7 & $16214077-4122218$ & -2.16 & -2.58 & -1.85 & III \\
\hline 8 & 16214973-4107017 & -2.06 & -2.20 & -2.03 & III \\
\hline 9 & $16215797-4131099$ & -2.33 & -2.37 & -2.38 & III \\
\hline 10 & $16222724-4100410$ & -2.26 & -2.61 & -2.07 & III \\
\hline 11 & $16222844-4113424$ & -2.24 & -2.45 & -2.08 & III \\
\hline 12 & $16222966-4123457$ & -1.53 & 0.33 & -1.89 & II \\
\hline 13 & $16223341-4103179$ & -2.07 & -2.36 & -1.79 & III \\
\hline 14 & $16230307-4021119$ & -2.00 & -2.12 & -1.93 & III \\
\hline 15 & 16231101-4117103 & -2.18 & -2.24 & -2.12 & III \\
\hline 16 & $16231476-4017151$ & -2.10 & -2.34 & -1.95 & III \\
\hline 17 & $16231598-4104009$ & -2.17 & -1.99 & -2.02 & III \\
\hline 18 & $16231844-4019439$ & -2.20 & -0.80 & -2.56 & III \\
\hline $19 *$ & $16232809-4015368$ & -1.59 & -2.70 & 0.05 & II \\
\hline 20 & $16233735-4014490$ & -2.29 & -2.67 & -2.11 & III \\
\hline 21 & $16234486-4107493$ & -1.80 & -1.79 & -2.02 & III \\
\hline 22 & $16234903-4026176$ & -1.92 & -2.09 & -2.03 & III \\
\hline 23 & $16235138-4010324$ & -2.26 & -2.36 & -2.36 & III \\
\hline 24 & $16242396-3952100$ & -2.22 & -2.36 & -2.17 & III \\
\hline 25 & $16242615-4011026$ & -2.21 & -2.32 & -2.28 & III \\
\hline 26 & $16244169-4004196$ & -2.32 & -2.61 & -2.19 & III \\
\hline 27 & $16244645-3956150$ & -1.12 & -1.34 & -0.83 & II \\
\hline 28 & $16245178-3956326$ & 0.22 & 0.46 & -0.36 & $\mathrm{~F}$ \\
\hline 29 & 16245564-3949147 & -2.13 & -1.85 & -2.28 & III \\
\hline 30 & $16245590-4011282$ & -2.05 & -1.83 & -2.21 & III \\
\hline 31 & $16245681-4008238$ & -2.21 & -1.64 & -2.44 & III \\
\hline 32 & $16255246-4018484$ & -2.23 & -2.05 & -2.18 & III \\
\hline 33 & $16255837-4009581$ & -2.10 & -2.33 & -1.84 & III \\
\hline 34 & $16260853-4017441$ & -2.34 & -2.28 & -2.39 & III \\
\hline 35 & 16261339-3949542 & -1.92 & -1.98 & -1.91 & III \\
\hline 36 & $16261365-4012186$ & -2.28 & -2.42 & -2.28 & III \\
\hline 37 & $16262551-3944472$ & -1.64 & -2.30 & -1.59 & III \\
\hline 38 & 16263114-3946153 & -2.14 & -2.55 & -1.01 & III \\
\hline 39 & $16263155-4004557$ & -2.26 & -2.34 & -2.29 & III \\
\hline 40 & $16263925-4015272$ & -1.49 & -1.47 & -1.56 & II \\
\hline 41 & 16265364-3954594 & -2.18 & -1.72 & -2.70 & III \\
\hline 42 & 16270309-3944139 & -2.01 & -1.81 & -2.40 & III \\
\hline 43 & 16273175-3956013 & -2.28 & -2.42 & -2.26 & III \\
\hline 44 & $16275054-3948100$ & -2.10 & -2.40 & -1.93 & III \\
\hline 45 & $16282647-3925428$ & -0.53 & -0.15 & -1.40 & II \\
\hline
\end{tabular}

*Transition object candidate. 
Table 8: Total number of YSO candidates in the Lupus V and VI clouds organized by Lada class. We also report for comparison the Merín et al. (2008) values for Lupus I, III and IV and the values for other clouds observed within the Spitzer c2d Survey. This last sample includes the statistics for Cha II (Alcalá et al. 2008), Perseus (Rebull et al. 2007; Jørgensen et al. 2008; Evans et al. 2009a), Serpens (Harvev et al. 2007b), and Ophiuchus (Padgett et al. 2008; Evans et al. 2009a).

\begin{tabular}{c|c|c|c|c|c|c}
\hline Lada Class & Lupus I $^{\dagger}$ & Lupus III $^{\dagger}$ & Lupus IV & Lupus V & Lupus VI & All c2d clouds $^{\ddagger}$ \\
\hline \hline I & $2(15 \%)$ & $2(3 \%)$ & $1(8 \%)$ & 0 & 0 & $165(16 \%)$ \\
Flat & $3(23 \%)$ & $6(9 \%)$ & $1(8 \%)$ & 0 & $1(2 \%)$ & $123(12 \%)$ \\
II & $6(47 \%)$ & $41(59 \%)$ & $5(42 \%)$ & $9(21 \%)$ & $5(11 \%)$ & $612(60 \%)$ \\
III & $2(15 \%)$ & $20(29 \%)$ & $5(42 \%)$ & $34(79 \%)$ & $39(87 \%)$ & $124(12 \%)$ \\
Total & 13 & 69 & 12 & 43 & 45 & 1024 \\
\hline
\end{tabular}

${ }^{\dagger}$ From Merín et al. (2008).

${ }^{\ddagger}$ From Evans et al. (2009a); see also their Table 5 for values relative to each c2d cloud.

Table 9: Summary of the clustering analysis versus Lada classes for Lupus V and VI (see Figure 14).

\begin{tabular}{|c|c|c|c|c|c|c|c|c|c|c|}
\hline Region & $\begin{array}{l}\text { Total YSO } \\
\text { candidates }\end{array}$ & Class I & Flat & Class II & Class III & $\begin{array}{l}\text { Cloud mass } \\
{\left[M_{\odot}\right]}\end{array}$ & $\begin{array}{l}\text { Volume } \\
{\left[\mathrm{pc}^{3}\right]}\end{array}$ & $\left\langle A_{V}\right\rangle$ & $\begin{array}{l}\mathrm{SFE}^{a} \\
{[\%]}\end{array}$ & $\begin{array}{l}\mathrm{SFE}^{b} \\
{[\%]}\end{array}$ \\
\hline$\overline{\mathrm{Va}}$ & 22 & 0 & 0 & 4 & 18 & 330 & 8.6 & 3.1 & 3.2 & 5.4 \\
\hline $\mathrm{Vb}$ & 13 & 0 & 0 & 3 & 10 & 210 & 4.9 & 2.9 & 2.9 & 5.0 \\
\hline VIa & 28 & 0 & 1 & 3 & 24 & 290 & 9.4 & 2.6 & 4.6 & 7.9 \\
\hline $\mathrm{VIb}$ & 15 & 0 & 0 & 1 & 14 & 160 & 3.5 & 2.8 & 4.4 & 7.1 \\
\hline
\end{tabular}

Table 10: Total cloud mass $\left(\mathrm{M}_{\text {cloud }}\right)$ above the star formation threshold ( $\mathrm{A}_{V} \approx 8.6 \mathrm{mag}$ ) and fraction of $\mathrm{M}_{\text {cloud }}$ below this threshold for the Lupus clouds observed with Spitzer.

\begin{tabular}{lll}
\hline \hline Region & $\begin{array}{l}\mathrm{M}_{\text {cloud }} \text { above } \\
\text { threshold }\left(\mathrm{M}_{\odot}\right)\end{array}$ & $\begin{array}{l}\text { Fraction of } \mathrm{M}_{\text {cloud }} \\
\text { below threshold }\end{array}$ \\
\hline Lupus I & 30 & $94 \%$ \\
Lupus III & 84 & $91 \%$ \\
Lupus IV & 47 & $75 \%$ \\
Lupus V & 0 & $100 \%$ \\
Lupus VI & 2.3 & $99 \%$ \\
\hline
\end{tabular}

Table 11: Number and properties of the OB stars within 1 pc of each Lupus cloud observed by Spitzer.

\begin{tabular}{lllllll}
\hline \hline Region & $\begin{array}{l}\text { N. of OB stars } \\
\text { within 1 pc }\end{array}$ & $\begin{array}{l}\text { RAJ2000 } \\
(\mathrm{hh:mm:ss})\end{array}$ & $\begin{array}{l}\text { DECJ2000 } \\
(\mathrm{dd}: \mathrm{mm}: \mathrm{ss})\end{array}$ & $\begin{array}{l}\text { Spectral } \\
\text { Type }\end{array}$ & $\begin{array}{l}\text { Luminosity } \\
(\mathrm{L} \odot)\end{array}$ & $\begin{array}{l}\text { Projected distance } \\
\text { to the cloud }\end{array}$ \\
\hline Lupus I & 1 & $15: 42: 41.0$ & $-34: 42: 36$ & $\mathrm{~B} 5$ & 163 & $36^{\prime}(0.65 \mathrm{pc})$ \\
Lupus III & 0 & - & - & - & - & - \\
Lupus IV & 0 & - & - & - & - & - \\
Lupus V & 1 & $16: 24: 31.8$ & $-37: 33: 58$ & $\mathrm{~B} 8$ & 119 & $4^{\prime}(0.96 \mathrm{pc})$ \\
Lupus VI & 1 & $16: 26: 20.5$ & $-39: 32: 27$ & $\mathrm{~B}$ & $2-3$ & $46^{\prime}(1.00 \mathrm{pc})$ \\
& & & & & & \\
\hline
\end{tabular}




\section{A. Appendix A}

In this section we describe three alternative methods for YSO classification that we used to confirm the Lada classification based on the computation of the single $\alpha_{K-24}$ SED slope (Sect. 4.1).

\section{A.1. Stellar to disk luminosity ratio}

The ratio between the stellar and the disk luminosity gives information on the evolutionary status of the disk, i.e. accreting, passive or debris disk (Kenyon \& Hartmann 1987).

We calculate the total luminosity $(\mathrm{L})$ of the each YSO candidate by integrating under all the available fluxes of the SED, as explained in Sect. [5.5. and the stellar luminosity $\left(\mathrm{L}_{\star}\right)$ by integrating under the best fitting NextGen photosphere model (Sect. 4.1); the difference between L and $\mathrm{L}_{\star}$ gives the disk luminosity $\left(\mathrm{L}_{\text {disk }}\right)$. Figure 17 shows that the disk fractional luminosities for the YSO candidates in Lupus V and VI is such that they are mainly surrounded by passive or debris disk, which confirms for most of them the Class III classification obtained from the $\alpha_{K-24}$-slope.

\section{A.2. Color-color and color-magnitude diagrams}

Infrared $\mathrm{CC}$ and $\mathrm{CM}$ diagrams are good diagnostic tools for investigating the evolutionary phase of circumstellar matter around YSOs (Hartmann et al. 2005; Lada et al. 2006).

Figure 18 shows CC and CM diagrams in the Spitzer and 2MASS bands for the YSO candidates in Lupus $\mathrm{V}$ and VI. The diagrams are compared with those derived from the grid of YSO models by Robitaille et al. (2006), adjusted in order to fit the distance of Lupus and matching the sensitivity cutoff of the 2MASS (Cutri et al. 2003) and Spitzer bands (Harvey et al. 2006; Jørgensen et al. 2006). This comparison shows that the majority of the YSO candidates in both Lupus V and VI have colors consistent with Stage III objects in the Robitaille et al. (2006) scheme, which is consistent with them being Class III objects in the Lada classification (see also Evans et al. 2009a). This cross-check endorses both our classification and the reliability of the Robitaille et al. (2006) grid of disk models.

\section{A.3. SED morphology parameters}

An interesting diagnostic to study disk evolution in young low-mass objects is the diagram of the SED excess slope, $\alpha_{\text {excess }}$, versus the wavelength at which the IR excess begins, $\lambda_{\text {turn }- \text { of } f}$ (see Cieza et al. 2007; Harvev et al. 2007b, 2008, for the definition of both parameters). Briefly, $\lambda_{\text {turn-off }}$ measures how far the circumstellar material extends inward to the central star, while $\alpha_{\text {excess }}$ measures how optically thick the disk is.

Figure 19 shows such diagram for our sample of YSO candidates in Lupus V and Lupus VI. Consistently with previous findings (i.e., Cieza et al. 2007; Harvev et al. 2008), the dispersion of $\alpha_{\text {excess }}$ starts increasing at $\lambda_{\text {turn-off }} \sim 4-5 \mu \mathrm{m}$, which corresponds to a distance of about $0.2 \mathrm{AU}$ from the central object under 


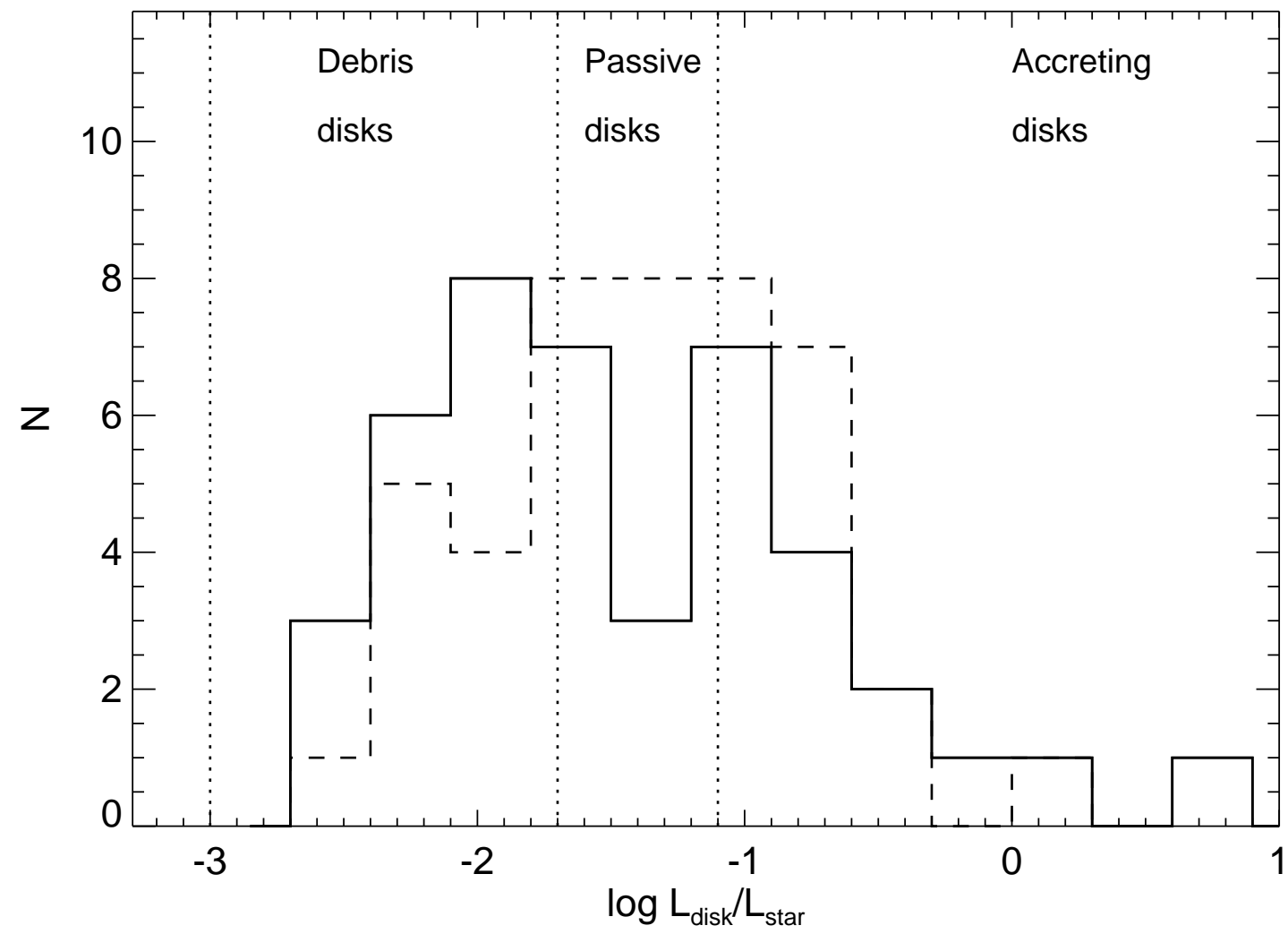

Fig. 17.- Distribution of disk fractional luminosities for YSO candidates in Lupus V (solid line) and Lupus VI (dashed line). The vertical dot-lines mark the typical boundaries of active, passive, and debris disks as defined by Kenyon \& Hartmann (1987) . 

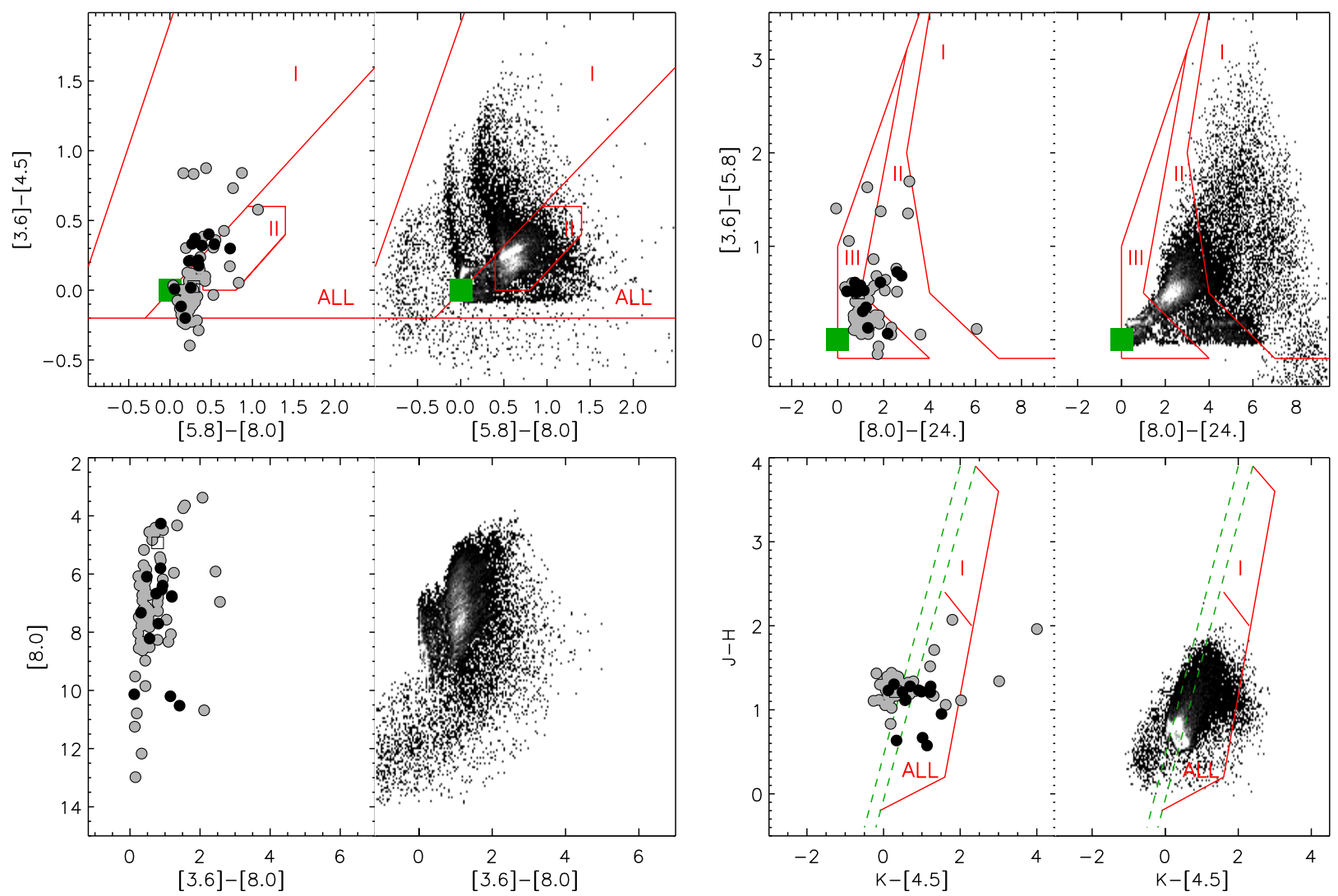

Fig. 18. - In each panel, the left sub-panel shows the CC or CM diagram for the combined YSO population in Lupus V and VI (black dots are the Class II YSO candidates, while gray dots ate the Class III YSO candidates), while the right sub-panel shows the colors derived from the SED models by Robitaille et al. (2006), in grayscale intensity representing the density of points. The areas corresponding to Stages I, II, and III as defined by Robitaille et al. (2006) are only shown in some sub-panels. The label "ALL" marks the regions where models of all evolutionary stages can be present. The big square and dashed-lines represent the location expected for normal and reddened photospheres, respectively. 
the assumption of thermal emission from a thin disk with blackbody grains (Alcalá et al. 2008). In other words, disks in later evolutionary phases (i.e. larger $\lambda_{\text {turn-off }}$ ) show a wider range of SED morphology (i.e. spread in $\left.\alpha_{\text {excess }}\right)$. The the majority of the YSO candidates in both Lupus V and VI have $\lambda_{\text {turn-off }} \gtrsim 4 \mu \mathrm{m}$ indicative of evolved disks. Again, this is consistent with them being mainly Class III objects. The two transitional object candidates (ID 3 in Lupus V and ID 19 in Lupus VI) lie in the upper-left region of this diagram, as expected for disks with large inner holes (Cieza et al. 2007). 


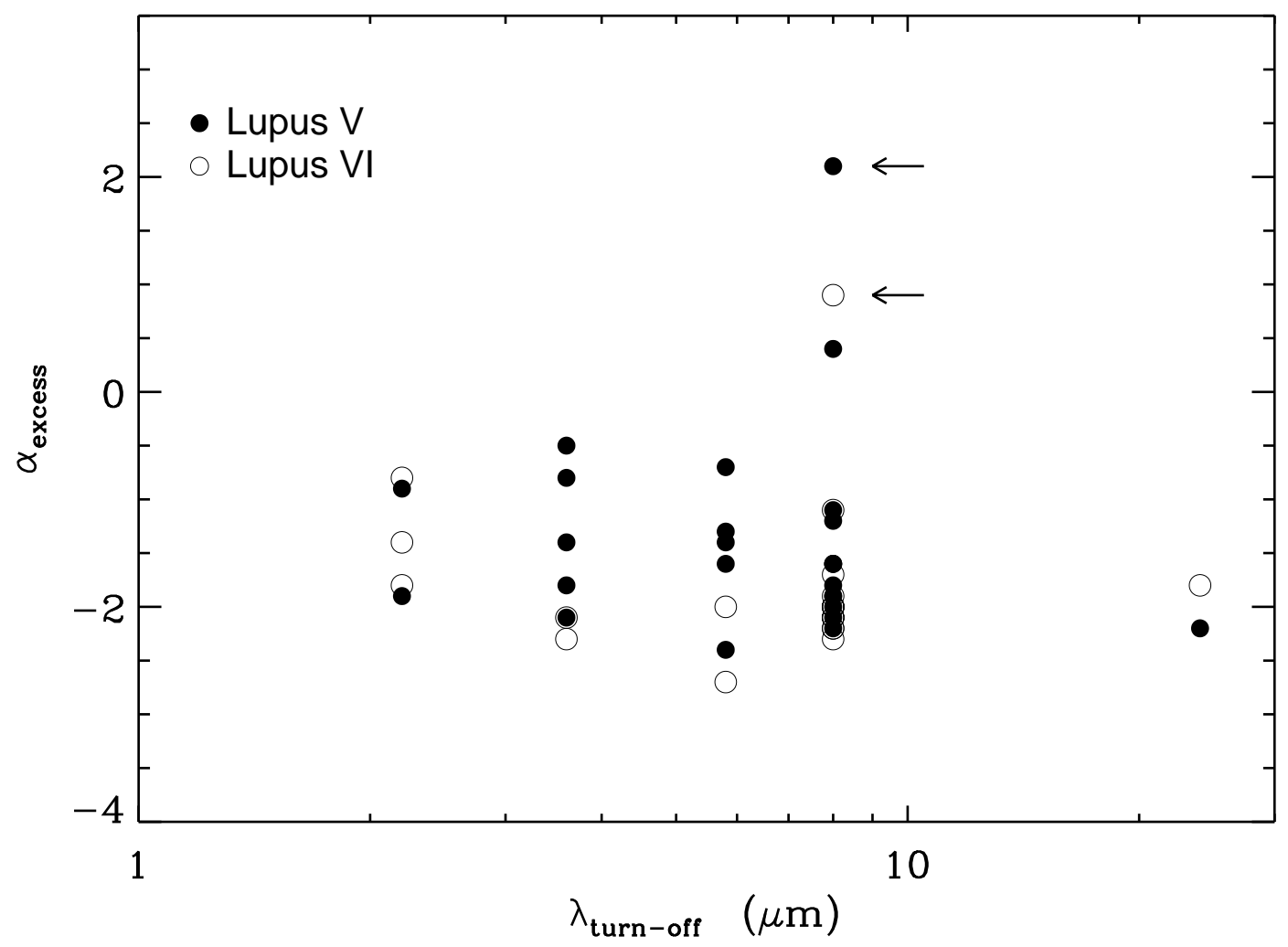

Fig. 19. - SED excess slopes $\left(\alpha_{\text {excess }}\right)$ as a function of the the wavelength at which the IR excess begins $\left(\lambda_{t u r n-o f f}\right)$ for the Lupus V and Lupus VI YSO candidates. The two arrows indicate the transitional object candidates. 


\section{REFERENCES}

Adams, F. C., Hollenbach, D., Laughlin, G., \& Gorti, U. 2004, ApJ, 611, 360

Alcalá, J. M., et al. 2008, ApJ, 676, 427

André, P., et al. 2010, A\&A, 518, L102

Balog, Z., Muzerolle, J., Rieke, G. H., Su, K. Y. L., Young, E. T., \& Megeath, S. T. 2007, ApJ, 660, 1532

Baraffe, I., Chabrier, G., Allard, F., \& Hauschildt, P. H. 1998, A\&A, 337, 403

Bouwman, J., Lawson, W. A., Dominik, C., Feigelson, E. D., Henning, T., Tielens, A. G. G. M., \& Waters, L. B. F. M. 2006, ApJ, 653, L57

Baraffe, I., \& Chabrier, G. 2010, A\&A, 521, A44

Basri, G., \& Reiners, A. 2006, AJ, 132, 663

Brandner, W., et al. 2000, AJ, 120, 950

Brown, J. M., et al. 2007, ApJ, 664, L107

Burns, J. A., Lamy, P. L., \& Soter, S. 1979, Icarus, 40,1

Cambrésy, L. 1999, A\&A, 345, 965

Casertano, S., \& Hut, P. 1985, ApJ, 298, 80

Chabrier, G. 2001, ApJ, 554, 1274

Chapman, N. L., et al. 2007, ApJ, 667, 288

Cieza, L., et al. 2007, ApJ, 667, 308

Cieza, L. A., et al. 2010, ApJ, 712, 925

Comerón, F., Fernández, M., Baraffe, I., Neuhäuser, R., \& Kaas, A. A. 2003, A\&A, 406, 1001

Comerón, F. 2008, Handbook of Star Forming Regions, Volume II, 295

Comerón, F., Spezzi, L., \& López Martí, B. 2009, A\&A, 500, 1045

Currie, T., Kenyon, S. J., Balog, Z., Rieke, G., Bragg, A., \& Bromley, B. 2008, ApJ, 672, 558

Currie, T., Plavchan, P., \& Kenyon, S. J. 2008, ApJ, 688, 597
Currie, T., Lada, C. J., Plavchan, P., Robitaille, T. P., Irwin, J., \& Kenyon, S. J. 2009, ApJ, 698,1

Cutri, R. M., et al. 2003, The IRSA 2MASS AllSky Point Source Catalog

D'Alessio, P., Calvet, N., Hartmann, L., Lizano, S., \& Cantó, J. 1999, ApJ, 527, 893

Dahm, S. E. 2005, AJ, 130, 1805

Duquennoy, A., \& Mayor, M. 1991, Bioastronomy: The Search for Extraterrestial Life - The Exploration Broadens, 390, 39

Enoch, M. L., Glenn, J., Evans, N. J., II, Sargent, A. I., Young, K. E., \& Huard, T. L. 2007, ApJ, 666,982

Evans, N. J., II, et al. 2003, PASP, 115, 965

Evans, II, N. J., Harvey, P. M., Dunham, M. M., et al., "Delivery Documentation for From Molecular Cores to Planet-forming Disks", http://ssc.spitzer.caltech.edu/legacy/original.html

Evans, N. J., et al. 2009, ApJS, 181, 321

Evans, N., et al. 2009, arXiv:0901.1691

Greene, T. P., Wilking, B. A., Andre, P., Young, E. T., \& Lada, C. J. 1994, ApJ, 434, 614

Guarcello, M. G., Micela, G., Damiani, F., Peres, G., Prisinzano, L., \& Sciortino, S. 2009, A\&A, 496, 453

Haisch, K. E., Jr., Lada, E. A., \& Lada, C. J. 2001, ApJ, 553, L153

Harvey, P. M., et al. 2006, ApJ, 644, 307

Harvey, P. M., et al. 2007, ApJ, 663, 1139

Harvey, P., Merín, B., Huard, T. L., Rebull, L. M., Chapman, N., Evans, N. J., II, \& Myers, P. C. 2007, ApJ, 663, 1149

Harvey, P. M., et al. 2008, ApJ, 680, 495

Hartmann, L., Megeath, S. T., Allen, L., Luhman, K., Calvet, N., D'Alessio, P., FrancoHernandez, R., \& Fazio, G. 2005, ApJ, 629, 88

Hauschildt, P. H., Allard, F., Ferguson, J., Baron, E., \& Alexander, D. R. 1999, ApJ, 525, 871 
Heiderman, A., Evans, N. J., II, Allen, L. E., Huard, T., \& Heyer, M. 2010, ApJ, 723, 1019

Hillenbrand, L. A. 2008, Physica Scripta Volume T, 130, 014024

Hughes, J., Hartigan, P., Krautter, J., \& Kelemen, J. 1994, AJ, 108, 1071

Johnstone, D., Hollenbach, D., \& Bally, J. 1998, ApJ, 499, 758

Johnstone, D., Di Francesco, J., \& Kirk, H. 2004, ApJ, 611, L45

Jørgensen, J. K., et al. 2006, ApJ, 645, 1246

Jørgensen, J. K., Johnstone, D., Kirk, H., Myers, P. C., Allen, L. E., \& Shirley, Y. L. 2008, ApJ, 683,822

Kenyon, S. J., \& Hartmann, L. 1987, ApJ, 323, 714

Kenyon, S. J., \& Hartmann, L. 1995, ApJS, 101, 117

Kim, K. H., et al. 2009, ApJ, 700, 1017

Knudsen, K. K., Walter, F., Weiss, A., Bolatto, A., Riechers, D. A., \& Menten, K. 2007, ApJ, 666,156

Kraus, A. L., \& Hillenbrand, L. A. 2008, ApJ, 686, L111

Kraus, A. L., \& Ireland, M. J. 2010, Highlights of Astronomy, 15, 766

Krautter, J. 1992, Low Mass Star Formation in Southern Molecular Clouds, 127

Krautter, J., Wichmann, R., Schmitt, J. H. M. M., Alcala, J. M., Neuhauser, R., \& Terranegra, L. 1997, A\&AS, 123, 329

Kroupa, P., \& Weidner, C. 2005, The Initial Mass Function 50 Years Later, 327, 175

Lada, C. J., \& Wilking, B. A. 1984, ApJ, 287, 610

Lada, C. J., \& Adams, F. C. 1992, ApJ, 393, 278

Lada, E. A., \& Lada, C. J. 1995, AJ, 109, 1682

Lada, C. J., \& Lada, E. A. 2003, ARA\&A, 41, 57

Lada, C. J. 2006, ApJ, 640, L63
Lada, C. J., et al. 2006, AJ, 131, 1574

Lada, C. J., Lombardi, M., \& Alves, J. F. 2010, arXiv:1009.2985

Makovoz, D., \& Marleau, F. R. 2005, PASP, 117, 1113

Mamajek, E. E., Meyer, M. R., Hinz, P. M., Hoffmann, W. F., Cohen, M., \& Hora, J. L. 2004, ApJ, 612, 496

Mason, B. D., Henry, T. J., Hartkopf, W. I., ten Brummelaar, T., \& Soderblom, D. R. 1998, AJ, 116,2975

Merín, B., et al. 2008, ApJS, 177, 551

Merín, B., et al. 2010, ApJ, 718, 1200

Miller, G. E., \& Scalo, J. M. 1979, ApJS, 41, 513

Monin, J.-L., Clarke, C. J., Prato, L., \& McCabe, C. 2007, Protostars and Planets V, 395

Muzerolle, J., Allen, L. E., Megeath, S. T., Hernández, J., \& Gutermuth, R. A. 2010, ApJ, 708, 1107

Natta, A., Testi, L., Muzerolle, J., Randich, S., Comerón, F., \& Persi, P. 2004, A\&A, 424, 603

Natta, A., Testi, L., \& Randich, S. 2006, A\&A, 452,245

Oliveira, I., et al. 2009, ApJ, 691, 672

Onishi, T., Mizuno, A., Kawamura, A., Ogawa, H., \& Fukui, Y. 1998, ApJ, 502, 296

Padgett, D. L., et al. 2008, ApJ, 672, 1013

Palla, F., Randich, S., Flaccomio, E., \& Pallavicini, R. 2005, ApJ, 626, L49

Plavchan, P., Jura, M., \& Lipscy, S. J. 2005, ApJ, 631,1161

Prato, L. 2007, ApJ, 657, 338

Raghavan, D., McAlister, H. A., Henry, T. J., \& Mason, B. D. 2007, IAU Symposium, 240, 254

Reed, B. C. 2003, AJ, 125, 2531

Rieke, G. H., et al. 2005, ApJ, 620, 1010 
Robitaille, T. P., Whitney, B. A., Indebetouw, R., Wood, K., \& Denzmore, P. 2006, ApJS, 167, 256

Robitaille, T. P., Whitney, B. A., Indebetouw, R., \& Wood, K. 2007, ApJS, 169, 328

Rebull, L. M., et al. 2007, ApJS, 171, 447

Robin, A. C., Reylé, C., Derrière, S., \& Picaud, S. 2003, A\&A, 409, 523

Sicilia-Aguilar, A., Hartmann, L. W., Fürész, G., Henning, T., Dullemond, C., \& Brandner, W. 2006, AJ, 132, 2135

Sicilia-Aguilar, A., Henning, T., \& Hartmann, L. W. 2010, ApJ, 710, 597

Skrutskie, M. F., Dutkevitch, D., Strom, S. E., Edwards, S., Strom, K. M., \& Shure, M. A. 1990, AJ, 99, 1187

Spezzi, L., et al. 2008, ApJ, 680, 1295

Störzer, H., \& Hollenbach, D. 1999, ApJ, 515, 669

Strom, K. M., Strom, S. E., Edwards, S., Cabrit, S., \& Skrutskie, M. F. 1989, AJ, 97, 1451

Tachihara, K., Toyoda, S., Onishi, T., Mizuno, A., Fukui, Y., \& Neuhäuser, R. 2001, PASJ, 53,1081

The, C. D. 2005, VizieR Online Data Catalog, 2263 ,

Teixeira, P. S., Lada, C. J., \& Alves, J. F. 2005, ApJ, 629, 276

Tothill, N. F. H., et al. 2009, ApJS, 185, 98

van Kempen, T. A., van Dishoeck, E. F., Salter, D. M., Hogerheijde, M. R., Jørgensen, J. K., \& Boogert, A. C. A. 2009, A\&A, 498, 167

Weingartner, J. C., \& Draine, B. T. 2001, ApJ, 548,296

Young, K. E., et al. 2005, ApJ, 628, 283

Zacharias, N., Monet, D. G., Levine, S. E., Urban, S. E., Gaume, R., \& Wycoff, G. L. 2005, VizieR Online Data Catalog, 1297, 0

This 2-column preprint was prepared with the AAS LATEX macros v5.2. 